- ARTICLES •

\title{
Convergence Error Estimates of the Crank-Nicolson Scheme for Solving Decoupled FBSDEs
}

\author{
Yang $\mathrm{Li}^{1}$, Jie Yang${ }^{2} \&$ Weidong Zhao ${ }^{2, *}$ \\ ${ }^{1}$ College of Science, University of Shanghai for Science and Technology, Shanghai 200093, China; \\ ${ }^{2}$ School of Mathematics, Shandong University, Jinan, Shandong 250100, China \\ Email:yangli@usst.edu.cn,yangjie218@mail.sdu.edu.cn,wdzhao@sdu.edu.cn
}

\begin{abstract}
The Crank-Nicolson (short for C-N) scheme for solving backward stochastic differential equation (BSDE), driven by Brownian motions, was first developed by the authors W. Zhao, L. Chen and S. Peng [SIAM J. Sci. Comput., 28 (2006), 1563-1581], and numerical experiments showed that the accuracy of this $\mathrm{C}-\mathrm{N}$ scheme was of second order for solving BSDE. This C-N scheme was extended to solve decoupled forwardbackward stochastic differential equations (FBSDEs) by W. Zhao, Y. Li and Y. Fu [Sci. China. Math., 57 (2014), 665-686], and it was numerically shown that the accuracy of the extended C-N scheme was also of second order. To our best knowledge, among all one-step (two-time level) numerical schemes with second-order accuracy for solving BSDE or FBSDEs, such as the ones in the above two papers and the one developed by the authors D. Crisan and K. Manolarakis [Ann. Appl. Probab., 24, 2 (2014), 652-678], the C-N scheme is the simplest one in applications. The theoretical proofs of second-order error estimates reported in the literature for these schemes for solving decoupled FBSDEs did not include the C-N scheme.

The purpose of this work is to theoretically analyze the error estimate of the C-N scheme for solving decoupled FBSDEs. Based on the Taylor and Itô-Taylor expansions, the Malliavin calculus theory (e.g., the multiple Malliavin integration-by-parts formula), and our new truncation error cancelation techniques, we rigorously prove that the strong convergence rate of the C-N scheme is of second order for solving decoupled FBSDEs, which fills the gap between the second-order numerical and theoretical analysis of the C-N scheme.
\end{abstract}

Keywords Convergence analysis, Crank-Nicolson scheme, decoupled forward backward stochastic differential equations, Malliavin calculus, trapezoidal rule.

$\operatorname{MSC}(\mathbf{2 0 1 0}) \quad 60 \mathrm{H} 35,65 \mathrm{C} 20$

\section{Introduction}

Let $(\Omega, \mathcal{F}, \mathbb{F}, P)$ be a filtered complete probability space, where $\mathbb{F}=\left(\mathcal{F}_{t}\right)_{0 \leqslant t \leqslant T}$ is the natural filtration of the standard $d$-dimensional Brownian motion $W_{t}=\left(W_{t}^{1}, \ldots, W_{t}^{d}\right)^{\top}, t \in[0, T]$, on the probability space $(\Omega, \mathcal{F}, \mathbb{F}, P)$, and $T$ is a fixed finite horizon. Let $L^{2}=L_{\mathcal{F}}^{2}(0, T)$ be the set of all $\mathcal{F}_{t^{-}}$adapted and mean-square-integrable vector or matrix processes for $t \in[0, T]$.

In this paper, on the space $(\Omega, \mathcal{F}, \mathbb{F}, P)$, we consider numerical solutions of decoupled forward-backward stochastic differential equations (FBSDEs) in the following integral form.

$$
\left\{\begin{array}{l}
X_{t}=X_{0}+\int_{0}^{t} b\left(s, X_{s}\right) d s+\int_{0}^{t} \sigma\left(s, X_{s}\right) d W_{s} \\
Y_{t}=\varphi\left(X_{T}\right)+\int_{t}^{T} f\left(s, X_{s}, Y_{s}, Z_{s}\right) d s-\int_{t}^{T} Z_{s} d W_{s}
\end{array}\right.
$$

* Corresponding author 
for $t \in[0, T]$, where $X_{0}$ is the initial condition of the forward stochastic differential equation (SDE), $\varphi\left(X_{T}\right)$ is the terminal condition of the backward stochastic differential equation (BSDE), $b$ is the drift coefficient valued in $\mathbb{R}^{d}, \sigma$ is the diffusion matrix valued in $\mathbb{R}^{d \times d}$, and $f$ valued in $\mathbb{R}$ is the generator function. Note that the two integrals with respect to $W_{s}$ in (1.1) are the Itô-type integrals.

A triple $\left(X_{s}, Y_{s}, Z_{s}\right):[0, T] \times \Omega \rightarrow \mathbb{R}^{d} \times \mathbb{R} \times \mathbb{R}^{1 \times d}$ is called an $L^{2}$-adapted solution of (1.1) if it is $\mathcal{F}_{s}$-adapted, $L^{2}$-integrable, and satisfies (1.1). In [19], under some standard conditions on the coefficients of (1.1), Pardoux and Peng originally proved the existence and uniqueness of the solution of nonlinear BSDE with more general terminal condition $Y_{T}=\xi \in \mathcal{F}_{T}$. And the solution $\left(Y_{s}, Z_{s}\right)$ of (1.1) can be represented as ( $[9,12,14,20,22])$

$$
Y_{s}=u\left(s, X_{s}\right), \quad Z_{s}=u_{x}\left(s, X_{s}\right) \sigma\left(s, X_{s}\right), \quad \forall s \in[0, T),
$$

where $u(t, x)$ is the smooth solution of the following parabolic partial differential equation (PDE).

$$
u_{t}(t, x)+\frac{1}{2} \sum_{i, j=1}^{d}\left[\sigma \sigma^{*}\right]_{i, j}(t, x) u_{x_{i} x_{j}}(t, x)+\sum_{i=1}^{d} b_{i}(t, x) u_{x_{i}}(t, x)+f\left(t, x, u(t, x), u_{x}(t, x) \sigma(t, x)\right)=0
$$

with the terminal condition $u(T, x)=\varphi(x)$.

FBSDEs have important applications in many fields including mathematical finance, partial differential equations, stochastic control, risk measure, and so on 1, 12, 17, 21, 23. So it is interesting and important to find solutions of FBSDEs. Usually, it is difficult to get the analytical solutions in an explicit closed form. Thus numerical methods for solving FBSDEs are desired, especially accurate, effective and efficient ones. Many numerical schemes for solving BSDE and decoupled FBSDEs have been developed, among which some are Euler-type methods with convergence rate $\frac{1}{2}$, such as [2, 4, 6, 8, 10, 11, 15, 24] and some are high-order numerical methods, such as [5, 16, 25, 32.

To our best knowledge in the literature, up to now, one-step second-order numerical schemes for solving BSDE and decoupled FBSDEs were proposed and studied in [5, 25, 28, 29, 32. In 2006, Zhao, Chen and Peng proposed numerical schemes for solving BSDE in [25, in which the Crank-Nicolson (short for C-N) is included. Numerical experiments showed that the accuracy of the C-N scheme was of second order for solving BSDE and its second-order convergence was theoretically proved in [28. And in 2014, Zhao, Li and Fu proposed three one-step second-order schemes, including the C-N scheme, for solving decoupled FBSDEs [29, and theoretically proved second-order convergence of them but not of the C-N one. By introducing new Gaussian processes, second-order numerical schemes were presented and analyzed for solving BSDE [5] and for decoupled FBSDEs in [32. The introduced new Gaussian processes simplified the proof of error estimates of the schemes, but doubled the computational complexity for solving BSDE or FBSDEs.

Among all these one-step second-order schemes, concerning their applications and coding in solving BSDE or FBSDEs, the simplest one is the C-N scheme. It was proposed in 25] for solving BSDE and the extension for solving decoupled FBSDEs was introduced in [29]. The second-order convergence rate of the C-N scheme for BSDE was proved in 28, but for decoupled FBSDEs is still open until now.

The purpose of this paper is to give a rigorously theoretical analysis on second-order convergence of the C-N scheme for solving decoupled FBSDEs (1.1). Compared with the proof in 28 for BSDE, the analysis for decoupled FBSDEs is much more difficult and complex. By the Taylor and Itô-Taylor expansions, the theory of multiple Malliavin calculus, and the error cancelation techniques, we are able to rigorously prove a general error estimate result for the C-N scheme, and based on this result, we finally obtained the theoretical second-order error estimate of the scheme for solving the decoupled FBSDEs.

Some notation to be used:

- $A^{\top}$ : the transpose of vector or matrix $A$.

- $|\cdot|$ : the norm for vector or matrix defined by $|A|^{2}=\operatorname{trace}\left(A^{\top} A\right)$.

- $C_{b}^{l, k, k, k}$ : the set of continuously differentiable functions $\psi:[0, T] \times \mathbb{R}^{d} \times \mathbb{R} \times \mathbb{R}^{d} \rightarrow \mathbb{R}$ with uniformly bounded partial derivatives $\partial_{t}^{l_{1}} \psi$ and $\partial_{x}^{k_{1}} \partial_{y}^{k_{2}} \partial_{z}^{k_{3}} \psi$ for $\frac{1}{2} \leqslant l_{1} \leqslant l$ and $1 \leqslant k_{1}+k_{2}+k_{3} \leqslant k$. Analogously we define $C_{b}^{l, k, k}$ and $C_{b}^{l, k}$.

- $C_{b}^{k}$ : the set of functions $\psi: x \in \mathbb{R}^{d} \rightarrow \mathbb{R}$ with uniformly bounded partial derivatives $\partial_{x}^{k_{1}} \psi$ for $1 \leqslant k_{1} \leqslant k$.

- $\mathcal{F}_{s}^{t, x}(t \leqslant s \leqslant T)$ : the $\sigma$-field generated by the diffusion process $\left\{X_{r}, t \leqslant r \leqslant s, X_{t}=x\right\}$.

- $\mathbb{E}_{s}^{t, x}[\eta]$ : the conditional mathematical expectation of the random variable $\eta$ under the $\sigma$-field $\mathcal{F}_{s}^{t, x}$, i.e., $\mathbb{E}_{s}^{t, x}[\eta]=\mathbb{E}\left[\eta \mid \mathcal{F}_{s}^{t, x}\right]$. Let $\mathbb{E}_{t}^{x}[\eta]=\mathbb{E}\left[\eta \mid \mathcal{F}_{t}^{t, x}\right]$. 
- $\partial_{x} \psi$ : the matrix valued function $\partial_{x} \psi=\left(\partial_{x^{j}} \psi^{i}\right)_{d \times d}(1 \leqslant i \leqslant d, 1 \leqslant j \leqslant d)$ for vector function $\psi=\left(\psi^{1}, \ldots, \psi^{d}\right)^{\top}$.

The rest of the paper is organized as follows. After we introduce some preliminaries in Section 2, we review the C-N scheme proposed in 29 for solving FBSDEs (1.1) in Section 3. Then we state our main error estimate results for the C-N scheme in Section 4, and prove them in Section 5. In Section 6, some conclusions are given.

\section{$2 \quad$ Preliminaries}

\subsection{Variational equations of the decoupled FBSDEs}

Let $\left(X_{r}^{t, x}, Y_{r}^{t, x}, Z_{r}^{t, x}\right)$ be the solution of the FBSDEs

$$
\left\{\begin{array}{l}
X_{r}^{t, x}=x+\int_{t}^{r} b\left(s, X_{s}^{t, x}\right) d s+\int_{t}^{r} \sigma\left(s, X_{s}^{t, x}\right) d W_{s}, \\
Y_{r}^{t, x}=\varphi\left(X_{T}^{t, x}\right)+\int_{r}^{T} f\left(s, X_{s}^{t, x}, Y_{s}^{t, x}, Z_{s}^{t, x}\right) d s-\int_{r}^{T} Z_{s}^{t, x} d W_{s},
\end{array}\right.
$$

for $r \in[t, T]$. Here the superscript ${ }^{t, x}$ means that the forward SDE starts from time $t$ at space point $x$.

Let $\nabla_{x_{i}} X_{r}^{t, x}$ and $\nabla_{x_{i}} Y_{r}^{t, x}$ be respectively the variation of $X_{r}^{t, x}$ and $Y_{r}^{t, x}$ with respect to (w.r.t.) $x_{i}$ which is the $i$-th component of $x=\left(x_{1}, \ldots, x_{d}\right)^{\top}$. Taking variation $\nabla_{x_{i}}$ on both sides of the equations in (2.1), we deduce

$$
\left\{\begin{array}{l}
\nabla_{x_{i}} X_{r}^{t, x}=e_{i}+\int_{t}^{r} b_{x}\left(s, X_{s}^{t, x}\right) \nabla_{x_{i}} X_{s}^{t, x} d s+\int_{t}^{r} \sigma_{x}^{j}\left(s, X_{s}^{t, x}\right) \nabla_{x_{i}} X_{s}^{t, x} d W_{s} \\
\nabla_{x_{i}} Y_{r}^{t, x}=\varphi_{x}\left(X_{T}^{t, x}\right) \nabla_{x_{i}} X_{T}^{t, x}+\int_{r}^{T} \nabla_{x_{i}} f\left(s, X_{s}^{t, x}, Y_{s}^{t, x}, Z_{s}^{t, x}\right) d s-\int_{r}^{T} \nabla_{x_{i}} Z_{s}^{t, x} d W_{s},
\end{array}\right.
$$

where $e_{i}=\overbrace{(0, \ldots, 0,1,0, \ldots, 0)}^{i}$ is the $i$-th coordinate basis vector of $\mathbb{R}^{d}, \sigma^{j}$ is the $j$-th column of $\sigma(\cdot)$, and

$$
\begin{aligned}
\nabla_{x} X_{s}^{t, x}= & {\left[\nabla_{x_{1}} X_{s}^{t, x}, \ldots, \nabla_{x_{d}} X_{s}^{t, x}\right]_{d \times d}, \quad \nabla_{x} Y_{s}^{t, x}=\left[\nabla_{x_{1}} Y_{s}^{t, x}, \ldots, \nabla_{x_{d}} Y_{s}^{t, x}\right]_{1 \times d}, } \\
\nabla_{x} Z_{s}^{t, x}= & {\left[\left[\nabla_{x_{1}} Z_{s}^{t, x}\right]^{\top}, \ldots,\left[\nabla_{x_{d}} Z_{s}^{t, x}\right]^{\top}\right]_{d \times d} } \\
\nabla_{x_{i}} f\left(s, X_{s}^{t, x}, Y_{s}^{t, x}, Z_{s}^{t, x}\right)= & f_{x}\left(s, X_{s}^{t, x}, Y_{s}^{t, x}, Z_{s}^{t, x}\right) \nabla_{x_{i}} X_{s}^{t, x}+f_{y}\left(s, X_{s}^{t, x}, Y_{s}^{t, x}, Z_{s}^{t, x}\right) \nabla_{x_{i}} Y_{s}^{t, x} \\
& +f_{z}\left(s, X_{s}^{t, x}, Y_{s}^{t, x}, Z_{s}^{t, x}\right) \nabla_{x_{i}} Z_{s}^{t, x}
\end{aligned}
$$

\subsection{The Itô-Taylor scheme for forward SDE}

For the time interval $[0, T]$, we first introduce the following time partition:

$$
0=t_{0}<\cdots<t_{N-1}<t_{N}=T
$$

with $\Delta=t_{n+1}-t_{n}$ for $n=0,1, \ldots, N-2$, and $t_{N}-t_{N-1}=\Delta^{2}$.

We shall call a row vector $\alpha=\left(j_{1}, j_{2}, \ldots, j_{l}\right)$ with $j_{i} \in\{0,1, \ldots, d\}$ for $i \in\{1,2, \ldots, l\}$, a multi-index of length $l:=l(\alpha) \in\{1,2, \ldots, d\}$, and denote by $v$ the multi-index of length zero $(l(v):=0)$. Let $\mathcal{M}$ be the set of all multi-indices, that is,

$$
\mathcal{M}=\left\{\left(j_{1}, j_{2}, \ldots, j_{l}\right): j_{i} \in\{0,1, \ldots, d\}, i \in\{1,2, \ldots, l\} \text { for } l=1,2, \ldots\right\} \cup\{v\} .
$$

Given a multi-index $\alpha \in \mathcal{M}$ with $l(\alpha) \geqslant 1$, we write $-\alpha$ and $\alpha$ - for the multi-index in $\mathcal{M}$ by deleting the first and last component of $\alpha$, respectively. Denote by $I_{\alpha}\left[g_{\alpha}(\cdot)\right]_{t_{n}, t_{n+1}}$ the multiple Itô integral recursively defined by

$$
I_{\alpha}\left[g_{\alpha}(\cdot)\right]_{t_{n}, t_{n+1}}= \begin{cases}X^{n}, & l=0, \\ \int_{t_{n}}^{t_{n+1}} I_{\alpha-}\left[g_{\alpha}(\cdot)\right]_{t_{n}, s} d s, & l \geqslant 1, j_{l}=0, \\ \int_{t_{n}}^{t_{n+1}} I_{\alpha-}\left[g_{\alpha}(\cdot)\right]_{t_{n}, s} d W_{s}^{j_{l}}, & l \geqslant 1, j_{l} \geqslant 1,\end{cases}
$$


where the Itô coefficient functions $g_{\alpha}(t, x)$ are defined by

$$
g_{\alpha}(t, x)= \begin{cases}x, & l=0 \\ g_{(0)}=b(t, x), \quad g_{(1)}=\sigma(t, x), & l=1 \\ L^{j_{1}} g_{-\alpha}, & l>1\end{cases}
$$

for all $(t, x) \in \mathbb{R} \times \mathbb{R}^{d}$, and $L^{j}$ are the differential operators defined by

$$
L^{0}=\partial_{t}+\sum_{k=1}^{d} b_{k} \frac{\partial}{\partial x_{k}}+\frac{1}{2} \sum_{k, l=1}^{d} \sum_{j=1}^{d} \sigma_{k j} \sigma_{l j} \frac{\partial^{2}}{\partial x_{k} \partial x_{l}} ; \quad L^{j}=\sum_{i=1}^{d} \sigma_{i j} \frac{\partial}{\partial x_{i}}, \quad 1 \leqslant j \leqslant d .
$$

In this paper, we will use the following weak order-2 Itô-Taylor schemes for solving SDE:

$$
X^{n+1}=\sum_{\alpha \in \Gamma_{2}} g_{\alpha}\left(t_{n}, X^{n}\right) I_{\alpha, n}=X^{n}+\phi^{n},
$$

where $X^{n}=\left(X_{1}^{n}, \ldots, X_{d}^{n}\right)^{\top}, \Gamma_{2}=\{\alpha \in \mathcal{M}: l(\alpha) \leqslant 2\}, I_{\alpha, n}:=I_{\alpha}[1]_{t_{n}, t_{n+1}}$ are the multiple Itô integrals for the index $\alpha$ over the time interval $\left[t_{n}, t_{n+1}\right]$, and $\phi^{n}=\left(\phi_{1}^{n}, \ldots, \phi_{d}^{n}\right)^{\top}$ with its $i$-th component

$$
\phi_{i}^{n}=b_{i} \Delta+\sum_{j_{1}=1}^{d} \sigma_{i j_{1}} I_{\left(j_{1}\right), n}+\sum_{j_{1}, j_{2}=1}^{d} L^{j_{1}} \sigma_{i j_{2}} I_{\left(j_{1}, j_{2}\right), n}+\frac{1}{2} \sum_{j_{1}=1}^{d}\left(L^{j_{1}} b_{i}+L^{0} \sigma_{i j_{1}}\right) \Delta I_{\left(j_{1}\right), n}+\frac{1}{2} L^{0} b_{i} \Delta^{2}
$$

with $I_{\left(j_{1}, j_{2}\right), n}=\int_{t_{n}}^{t_{n+1}} \int_{t_{n}}^{s_{2}} d W_{s_{1}}^{j_{1}} d W_{s_{2}}^{j_{2}}$ for $j_{1}, j_{2} \in\{1, \ldots, d\}$. In the sequel, if there is no confusion, for a function $a=a(t, x)$, we denote $a\left(t_{n}, X^{n}\right)$ by $a$.

\subsection{The Malliavin calculus on SDE and BSDE}

Suppose that $H$ is a real separable Hilbert space with scalar product denoted by $\langle\cdot, \cdot\rangle_{H}$. The norm of an element $h \in H$ will be denoted by $\|h\|_{H}$. Let $\mathcal{W}=\{W(h), h \in H\}$ denote an isonormal Gaussian process associated with the Hilbert space $H$ on $(\Omega, \mathcal{F}, \mathbb{F}, P)$.

For the Brownian motion $W_{t}=\left(W_{t}^{1}, \ldots, W_{t}^{d}\right)^{\top}$, we define a random variable of the form $F=$ $f\left(W\left(h^{1}\right), \ldots, W\left(h^{d}\right)\right)$, where $h^{k}=\left(h^{k, 1}, \ldots, h^{k, d}\right)$ and

$$
W\left(h^{k}\right)=\int_{0}^{\infty} h_{t}^{k} d W_{t}, \quad 1 \leqslant k \leqslant d .
$$

Similarly we define $W^{i}\left(h^{k, i}\right)=\int_{0}^{\infty} h_{t}^{k, i} d W_{t}^{i}$, then it is easy to see $W\left(h^{k}\right)=\sum_{i=1}^{d} W^{i}\left(h^{k, i}\right)$. For the index $\alpha=(i)(0 \leqslant i \leqslant d)$, let $D_{t}^{\alpha}=D_{i, t}(0 \leqslant t \leqslant T)$ be the Malliavin derivative of order one w.r.t. $W_{s}^{i}$, with the convention that $D_{0, t}$ is just the identity, i.e., $D_{0, t}=1$ and

$$
D_{i, t} F=\sum_{k=1}^{d} \frac{\partial f}{\partial x^{k, i}}\left(W\left(h^{1}\right), \ldots, W\left(h^{d}\right)\right) h_{t}^{k, i}, \quad \text { for } \quad i=1, \ldots, d .
$$

From an intuitive point of view $D_{i, t} F$ represents the derivative of $F$ w.r.t. the increment of $i$-th Brownian motion $W^{i}$ corresponding to $t$. We will sometimes use the following intuitive notation

$$
D_{0, t}=1, \quad D_{i, t} F=\frac{\partial F}{\partial \Delta_{t}^{i}},
$$

where $\Delta_{t}^{i}=\Delta W_{n}^{i}=W_{t_{n+1}}^{i}-W_{t_{n}}^{i}$ for $1 \leqslant i \leqslant d$ and $t_{n} \leqslant t \leqslant t_{n+1}$. For a random variable $X=$ $\left(X^{1}, \ldots, X^{d}\right)^{\top}$, we assume

$$
D_{i, t} X=\left(D_{i, t} X^{1}, \ldots, D_{i, t} X^{d}\right)^{\top}, \quad D_{t} X=\left(D_{1, t} X, \ldots, D_{d, t} X\right)_{d \times d}
$$

and

$$
D_{s_{1} \cdots s_{l}}^{\alpha}=D_{s_{1} \cdots s_{l}}^{\left(j_{1}, \ldots, j_{l}\right)}=D_{j_{1}, s_{1}}, \cdots D_{j_{l}, s_{l}}
$$


for the multi-indices $\alpha=\left(j_{1}, \ldots, j_{l}\right) \in \mathcal{A}_{l}$ with $j_{i} \in\{0,1, \cdots, d\}(i=1, \ldots, l)$, where $\mathcal{A}_{l}=\{\alpha \in \mathcal{M}$ :

$l(\alpha)=l\}$, and $s_{i} \in[0, T]$. For any integer $p \geqslant 1, \mathbb{D}^{k, p}$ is the domain of $\mathbb{D}^{k}(k \in \mathbb{N})$ in $L^{p}(\Omega)$, that is, $\mathbb{D}^{k, p}$ is the closure of the class of smooth random variables $F$ w.r.t. the norm

$$
\|F\|_{k, p}^{p}=\mathbb{E}\left[|F|^{p}\right]+\sum_{j=1}^{k} \sum_{|\alpha|=l} \int_{0}^{T} \ldots \int_{0}^{T} \mathbb{E}\left[\left|D_{s_{1}, \ldots, s_{l}}^{\alpha} F\right|^{p}\right] d s_{1} \ldots d s_{l} .
$$

For $p=2$, the space $\mathbb{D}^{1,2}$ is a Hilbert space with the scalar product

$$
\langle F, G\rangle=\mathbb{E}[F G]+\mathbb{E}\left[\langle D F, D G\rangle_{H}\right]
$$

where $\langle D F, D G\rangle_{H}:=\int_{0}^{T} \sum_{i=1}^{d} D_{i, t} F D_{i, t} G d t$.

For $t_{n}<r \leqslant s \leqslant t \leqslant t_{n+1}$ and $i, j, k \in\{1,2, \ldots, d\}$, by taking the Malliavin derivative $D_{j, t}, D_{i, s} D_{j, t}$ and $D_{k, r} D_{i, s} D_{j, t}$ to the multiple integral $I_{\left(j_{1}, j_{2}\right), n}$, we easily get

$$
\begin{aligned}
D_{j, t} I_{\left(j_{1}, j_{2}\right), n} & =\left(\int_{t_{n}}^{t} d W_{s_{1}}^{j_{1}}\right) \delta_{j j_{2}}+\int_{t}^{t_{n+1}} D_{j, t}\left(W_{s_{2}}^{j_{1}}-W_{t}^{j_{1}}+W_{t}^{j_{1}}-W_{t_{n}}^{j_{1}}\right) d W_{s_{2}}^{j} \\
& =\left(W_{t}^{j_{1}}-W_{t_{n}}^{j_{1}}\right) \delta_{j j_{2}}+\left(W_{t_{n+1}}^{j_{2}}-W_{t}^{j_{2}}\right) \delta_{j j_{1}}, \\
D_{i, s} D_{j, t} I_{\left(j_{1}, j_{2}\right), n} & =D_{i, s}\left(W_{t}^{j_{1}}-W_{t_{n}}^{j_{1}}\right) \delta_{j j_{2}}+D_{i, s}\left(W_{t_{n+1}}^{j_{2}}-W_{t}^{j_{2}}\right) \delta_{j j_{1}}=\delta_{i j_{1}} \delta_{j j_{2}}, \\
D_{k, r} D_{i, s} D_{j, t} I_{\left(j_{1}, j_{2}\right), n} & =0 .
\end{aligned}
$$

Then for $s_{1}<s_{2}<s_{3}$ and $\alpha=\left(j_{1}, j_{2} \cdot j_{3}\right)$, it holds that

$$
\begin{aligned}
& D_{s_{1} s_{2} s_{3}}^{\alpha} \phi_{i}^{n}=D_{j_{3}, s_{3}} \phi_{i}^{n}= \sigma_{i j_{3}}+\sum_{j=1}^{d} L^{j} \sigma_{i j_{3}}\left(W_{s_{3}}^{j}-W_{t_{n}}^{j}\right)+\sum_{j=1}^{d} L^{j_{3}} \sigma_{i j}\left(W_{t_{n+1}}^{j}-W_{s_{3}}^{j}\right) \\
&+\frac{1}{2}\left(L^{j_{3}} b_{i}+L^{0} \sigma_{i j_{3}}\right) \Delta, \quad j_{1}=j_{2}=0, \quad j_{3} \geqslant 1, \\
& D_{s_{1} s_{2} s_{3}}^{\alpha} \phi_{i}^{n}=D_{s_{2}, j_{2}} D_{s_{3}, j_{3}} \phi_{i}^{n}=\sum_{j=1}^{d} L^{j} \sigma_{i j_{3}} \delta_{j j_{2}}=L^{j_{2}} \sigma_{i j_{3}}, \quad j_{1}=0, \quad j_{2}, j_{3} \geqslant 1, \\
& D_{s_{1} s_{2} s_{3}}^{\alpha} \phi_{i}^{n}=D_{j_{1}, s_{1}} D_{j_{2}, s_{2}} D_{j_{3}, s_{3}} \phi_{i}^{n}=0, \quad j_{1}, j_{2}, j_{3} \geqslant 1 .
\end{aligned}
$$

For the Malliavin derivative operator $D_{t}$, we introduce the following two lemmas.

Lemma 2.3.1 (Integration-by-parts formula). For $F \in \mathbb{D}^{1,2}, u \in L^{2}(\Omega ; H)$ and $i, j \in\{1,2, \ldots, d\}$, we have

$$
\begin{aligned}
& \mathbb{E}\left[F \int_{0}^{T} u_{t} d W_{t}^{i}\right]=\mathbb{E}\left[\int_{0}^{T} D_{i, t} F u_{t} d t\right], \quad D_{i, t} \int_{0}^{T} u_{s} d s=\int_{t}^{T} D_{i, t} u_{s} d s, \\
& D_{i, t} \int_{0}^{T} u_{s} d W_{s}^{j}=u_{t} \delta_{i j}+\int_{t}^{T} D_{i, t} u_{s} d W_{s}^{j}, \quad 0<t<T,
\end{aligned}
$$

where $\delta_{i j}$ is the Kronecker delta function [18].

Lemma 2.3.2 (Chain-rule). If $\psi: \mathbb{R}^{m} \rightarrow \mathbb{R}$ is a continuously differential function with bounded partial derivatives, $F=\left(F_{1}, \ldots, F_{m}\right)\left(F_{i} \in \mathbb{D}^{1,2}\right)$ is a random vector, and the solution $\left(X_{r}^{t, x}, Y_{r}^{t, x}, Z_{r}^{t, x}\right)$ of (2.1) is in $\mathbb{D}^{1,2}$, then the following identities hold [12, 18]

$$
\begin{aligned}
& D_{i, t} \psi(F)=\sum_{j=1}^{d} \frac{\partial \psi}{\partial x_{j}}(F) D_{i, t} F_{j}, \\
& D_{s} X_{r}^{t, x} I_{s \leqslant r}=\nabla_{x} X_{r}^{t, x}\left(\nabla_{x} X_{s}^{t, x}\right)^{-1} \sigma\left(s, X_{s}^{t, x}\right) I_{s \leqslant r}, \quad D_{s} Y_{r}^{t, x} I_{s \leqslant r}=\nabla_{x} Y_{r}^{t, x} D_{s} X_{r}^{t, x} I_{s \leqslant r} .
\end{aligned}
$$

\section{The C-N Scheme for solving decoupled FBSDEs}

Let $\left(X_{t}^{t_{n}, X^{n}}, Y_{t}^{t_{n}, X^{n}}, Z_{t}^{t_{n}, X^{n}}\right)_{t_{n} \leqslant t \leqslant T}(0 \leqslant n \leqslant N-1)$ be the solution of the FBSDEs (2.1) with $t$ and $x$ replaced by $t_{n}$ and $X^{n}$, respectively. Then we have

$$
Y_{t_{n}}^{t_{n}, X^{n}}=Y_{t_{n+1}}^{t_{n}, X^{n}}+\int_{t_{n}}^{t_{n+1}} f\left(s, X_{s}^{t_{n}, X^{n}}, Y_{s}^{t_{n}, X^{n}}, Z_{s}^{t_{n}, X^{n}}\right) d s-\int_{t_{n}}^{t_{n+1}} Z_{s}^{t_{n}, X^{n}} d W_{s} .
$$


By taking the conditional mathematical expectation $\mathbb{E}_{t_{n}}^{X^{n}}[\cdot]$ to the above equation gives

$$
Y_{t_{n}}^{t_{n}, X^{n}}=\mathbb{E}_{t_{n}}^{X^{n}}\left[Y_{t_{n+1}}^{t_{n}, X^{n}}\right]+\int_{t_{n}}^{t_{n+1}} \mathbb{E}_{t_{n}}^{X^{n}}\left[f_{s}^{t_{n}, X^{n}}\right] d s
$$

with $f_{s}^{t_{n}, X^{n}}:=f\left(s, X_{s}^{t_{n}, X^{n}}, Y_{s}^{t_{n}, X^{n}}, Z_{s}^{t_{n}, X^{n}}\right)$. When $n=N-1$, we use the Euler method to approximate the integral in (3.2) and obtain

$$
Y_{t_{N-1}}^{t_{N-1}, X^{N-1}}=\mathbb{E}_{t_{N-1}}^{X^{N-1}}\left[\varphi\left(X^{N}\right)\right]+\Delta^{2} f_{t_{N-1}}^{t_{N-1}, X^{N-1}}+\sum_{j=1}^{2} R_{y j}^{N-1}
$$

where

$$
R_{y 1}^{N-1}=\int_{t_{N-1}}^{t_{N}}\left\{\mathbb{E}_{t_{N-1}}^{X^{N-1}}\left[f_{s}^{t_{N-1}, X^{N-1}}\right]-f_{t_{N-1}}^{t_{N-1}, X^{N-1}}\right\} d s, \quad R_{y 2}^{N-1}=\mathbb{E}_{t_{N-1}}^{X^{N-1}}\left[\varphi\left(X_{t_{N}}^{t_{N-1}, X^{N-1}}\right)-\varphi\left(X^{N}\right)\right] .
$$

For $0 \leqslant n \leqslant N-2$, by using the trapezoidal rule to approximate the integral in (3.2), we deduce

$$
Y_{t_{n}}^{t_{n}, X^{n}}=\mathbb{E}_{t_{n}}^{X^{n}}\left[Y_{t_{n+1}}^{t_{n+1}, X^{n+1}}\right]+\frac{1}{2} \Delta f_{t_{n}}^{t_{n}, X^{n}}+\frac{1}{2} \Delta \mathbb{E}_{t_{n}}^{X^{n}}\left[f_{t_{n+1}}^{t_{n+1}, X^{n+1}}\right]+\sum_{j=1}^{2} R_{y j}^{n},
$$

where

$$
\begin{aligned}
& R_{y 1}^{n}=\int_{t_{n}}^{t_{n+1}}\left\{\mathbb{E}_{t_{n}}^{X^{n}}\left[f_{s}^{t_{n}, X^{n}}\right]-\frac{1}{2} \mathbb{E}_{t_{n}}^{X^{n}}\left[f_{t_{n+1}}^{t_{n}, X^{n}}\right]-\frac{1}{2} f_{t_{n}}^{t_{n}, X^{n}}\right\} d s, \\
& R_{y 2}^{n}=\mathbb{E}_{t_{n}}^{X^{n}}\left[Y_{t_{n+1}}^{t_{n}, X^{n}}-Y_{t_{n+1}}^{t_{n+1}, X^{n+1}}\right]+\frac{1}{2} \Delta \mathbb{E}_{t_{n}}^{X^{n}}\left[f_{t_{n+1}}^{t_{n}, X^{n}}-f_{t_{n+1}}^{t_{n+1}, X^{n+1}}\right] .
\end{aligned}
$$

Let $\Delta W_{n}=W_{t_{n+1}}-W_{t_{n}}$ for $0 \leqslant n \leqslant N-1$. Multiplying (3.1) by $\Delta W_{n}^{\top}$, taking the conditional mathematical expectation $\mathbb{E}_{t_{n}}^{X^{n}}[\cdot]$ on both sides of the derived equation, and then using the Itô isometry formula we obtain

$$
-\mathbb{E}_{t_{n}}^{X^{n}}\left[Y_{t_{n+1}}^{t_{n}, X^{n}} \Delta W_{n}^{\top}\right]=\int_{t_{n}}^{t_{n+1}} \mathbb{E}_{t_{n}}^{X^{n}}\left[f_{s}^{t_{n}, X^{n}} \Delta W_{n}^{\top}\right] d s-\int_{t_{n}}^{t_{n+1}} \mathbb{E}_{t_{n}}^{X^{n}}\left[Z_{s}^{t_{n}, X^{n}}\right] d s .
$$

When $n=N-1$, the Euler scheme is applied to approximate the integral in the above equation, then

$$
Z_{t_{N-1}}^{t_{N-1}, X^{N}-1}=\frac{1}{\Delta^{2}} \mathbb{E}_{t_{N-1}}^{X^{N-1}}\left[\varphi\left(X^{N}\right) \Delta W_{N-1}^{\top}\right]+\frac{1}{\Delta^{2}} \sum_{j=1}^{2} R_{z j}^{N-1}
$$

where

$$
\begin{aligned}
& R_{z 1}^{N-1}=\int_{t_{N-1}}^{t_{N}} \mathbb{E}_{t_{N-1}}^{X^{N-1}}\left[f_{s}^{t_{N-1}, X^{N-1}} \Delta W_{N-1}^{\top}\right] d s-\int_{t_{N-1}}^{t_{N}} \mathbb{E}_{t_{N-1} X^{N-1}}^{\top}\left(\left[Z_{s}^{t_{N-1}, X^{N-1}}\right]-Z_{t_{N-1}}^{t_{N-1}, X^{N-1}}\right) d s, \\
& R_{z 2}^{N-1}=\mathbb{E}_{t_{N-1}^{X}}^{X^{N-1}}\left[\varphi\left(X_{t_{N}, X^{N-1}}^{t_{N-1}}\right) \Delta W_{N-1}^{\top}-\varphi\left(X^{N}\right) \Delta W_{N-1}^{\top}\right] .
\end{aligned}
$$

For $0 \leqslant n \leqslant N-2$, following similar derivation of the equation (3.4), we obtain the second reference equation as

$$
\frac{1}{2} \Delta Z_{t_{n}}^{t_{n}, X^{n}}=-\frac{1}{2} \Delta \mathbb{E}_{t_{n}}^{X^{n}}\left[Z_{t_{n+1}}^{t_{n+1}, X^{n+1}}\right]+\mathbb{E}_{t_{n}}^{X^{n}}\left[Y_{t_{n+1}}^{t_{n+1}, X^{n+1}} \Delta W_{n}^{\top}\right]+\frac{1}{2} \Delta \mathbb{E}_{t_{n}}^{X^{n}}\left[f_{t_{n+1}}^{t_{n+1}, X^{n+1}} \Delta W_{n}^{\top}\right]+\sum_{j=1}^{2} R_{z j}^{n},
$$

where

$$
\begin{aligned}
R_{z 1}^{n}= & \int_{t_{n}+1}^{t_{n+1}} \mathbb{E}_{t_{n}}^{X^{n}}\left[f_{s}^{t_{n}, X^{n}} \Delta W_{n}^{\top}\right] d s-\frac{1}{2} \Delta \mathbb{E}_{t_{n}}^{X^{n}}\left[f_{t_{n+1}}^{t_{n}, X^{n}} \Delta W_{n}^{\top}\right] \\
& -\int_{t_{n}}^{t_{n+1}}\left\{\mathbb{E}_{t_{n}}^{X^{n}}\left[Z_{s}^{t_{n}, X^{n}}\right]-\frac{1}{2} \mathbb{E}_{t_{n}}^{X_{n}}\left[Z_{t_{n}, X^{n}}^{t_{n}, X^{n}}\right]-\frac{1}{2} Z_{t_{n}}^{t_{n}, X^{n}}\right\} d s, \\
R_{z 2}^{n}= & -\frac{1}{2} \Delta \mathbb{E}_{t_{n}}^{X^{n}}\left[Z_{t_{n+1}, X^{n}}^{t_{n}, X_{t_{n+1}}, X^{n+1}}\right]+\mathbb{E}_{t_{n}}^{t_{n}}\left[\left(Y_{t_{n+1}, X^{n}}^{t_{n}}-Y_{t_{n+1}}^{t_{n+1}, X^{n+1}}\right) \Delta W_{n}^{\top}\right] \\
& +\frac{1}{2} \Delta \mathbb{E}_{t_{n}}^{X^{n}}\left[\left(f_{t_{n+1}}^{t_{n}, X^{n}}-f_{t_{n+1}}^{t_{n+1}, X^{n+1}}\right) \Delta W_{n}^{\top}\right] .
\end{aligned}
$$

Let $\left(Y^{n}, Z^{n}\right)$ denote the approximation to the exact solution $\left(Y_{t_{n}}^{t_{n}, X^{n}}, Z_{t_{n}}^{t_{n}, X^{n}}\right)$ of BSDE (3.1) for $n=N-1, \ldots, 0$. For simple representation, we denote $f^{n}:=f\left(t_{n}, X^{n}, Y^{n}, Z^{n}\right)$. Now, based on the reference equations (3.3), (3.4), (3.6) and (3.7), we introduce the Crank-Nicolson scheme (Scheme 2.1 proposed in 29]) for solving decoupled FBSDEs (1.1). 
Scheme 3.1. Suppose that the initial condition $X_{0}$ for the forward SDE in (1.1) and the terminal condition $\varphi$ for the BSDE in (1.1) are given.

1. For $n=N-1, t_{N}-t_{N-1}=\Delta^{2}$, solve $X^{N}, Y^{N-1}$ and $Z^{N-1}$ by

$$
\begin{aligned}
X^{N} & =X^{N-1}+b\left(t_{N-1}, X^{N-1}\right) \Delta^{2}+\sigma\left(t_{N-1}, X^{N-1}\right) \Delta W_{N-1}^{\top}, \\
Z^{N-1} & =\frac{1}{\Delta^{2}} \mathbb{E}_{t_{N-1}}^{X^{N-1}}\left[Y^{N} \Delta W_{N-1}^{\top}\right], \\
Y^{N-1} & =\mathbb{E}_{t_{N-1} X^{N-1}}^{\top}\left[Y^{N}\right]+\Delta^{2} f^{N-1} .
\end{aligned}
$$

2. For $n=N-2, \ldots, 0$, solve $X^{n+1}, Y^{n}, Z^{n}$ by

$$
\begin{aligned}
X^{n+1} & =X^{n}+\sum_{\alpha \in \Gamma_{2} \backslash\{v\}} g_{\alpha}\left(t_{n}, X^{n}\right) I_{\alpha, n}, \\
\frac{1}{2} \Delta Z^{n} & =-\frac{1}{2} \Delta \mathbb{E}_{t_{n}}^{X^{n}}\left[Z^{n+1}\right]+\mathbb{E}_{t_{n}}^{X^{n}}\left[Y^{n+1} \Delta W_{n}^{\top}\right]+\frac{1}{2} \Delta \mathbb{E}_{t_{n}}^{X^{n}}\left[f^{n+1} \Delta W_{n}^{\top}\right], \\
Y^{n} & =\mathbb{E}_{t_{n}}^{X^{n}}\left[Y^{n+1}\right]+\frac{1}{2} \Delta f^{n}+\frac{1}{2} \Delta \mathbb{E}_{t_{n}}^{X^{n}}\left[f^{n+1}\right] .
\end{aligned}
$$

Remark 3.1. 1. In 2006, the authors in 25] proposed the following scheme for solving BSDE.

$$
\begin{aligned}
Y^{n} & =\mathbb{E}_{t_{n}}^{x}\left[Y^{n+1}\right]+\Delta t_{n}\left[\left(1-\theta_{1}^{n}\right) \mathbb{E}_{t_{n}}^{x}\left[f^{n+1}\right]+\theta_{1}^{n} f^{n}\right], \\
0 & =\mathbb{E}_{t_{n}}^{x}\left[Y^{n+1} \Delta W_{n}^{\top}\right]+\Delta t_{n}\left(1-\theta_{2}^{n}\right) \mathbb{E}_{t_{n}}^{x}\left[f^{n+1} \Delta W_{n}^{\top}\right]-\Delta t_{n}\left\{\left(1-\theta_{2}^{n}\right) \mathbb{E}_{t_{n}}^{x}\left[Z^{n+1}\right]+\theta_{2}^{n} Z^{n}\right\},
\end{aligned}
$$

where $\Delta t_{n}=t_{n+1}-t_{n}$ and $f^{n}=f\left(t_{n}, Y^{n}, Z^{n}\right)$ for $n=N-1, \ldots, 0$ with the parameters $\theta_{1}^{n}$ and $\theta_{2}^{n}$ in $[0,1]$. The C-N scheme for BSDE is the case $\theta_{1}^{n}=\theta_{2}^{n}=\frac{1}{2}$. The second-order error estimate results were proved in 28 .

2. In 2012, the authors in 27] developed the following $\theta$-scheme for BSDE: Given the terminal values $Y^{N}$ and $Z^{N}$, solve $Y^{n}$ and $Z^{n}$ by

$$
\begin{aligned}
Y^{n} & =\mathbb{E}_{t_{n}}^{x}\left[Y^{n+1}\right]+\Delta t_{n}\left[\left(1-\theta_{1}\right) \mathbb{E}_{t_{n}}^{x}\left[f^{n+1}\right]+\theta_{1} f^{n}\right], \\
\theta_{3} \Delta t_{n} Z^{n} & =\theta_{4} \Delta t_{n} \mathbb{E}_{t_{n}}^{x}\left[Z^{n+1}\right]+\left(\theta_{3}-\theta_{4}\right) \mathbb{E}_{t_{n}}^{x}\left[Y^{n+1} \Delta W_{n}^{\top}\right]+\left(1-\theta_{2}\right) \Delta t_{n} \mathbb{E}_{t_{n}}^{x}\left[f^{n+1} \Delta W_{n}^{\top}\right],
\end{aligned}
$$

where $\Delta t_{n}=t_{n+1}-t_{n}$ and $f^{n}=f\left(t_{n}, Y^{n}, Z^{n}\right)$ for $n=N-1, \ldots, 0$ with the deterministic parameters $\theta_{i} \in[0,1](i=1,2), \theta_{3} \in(0,1]$, and $\theta_{4} \in[-1,1]$ constrained by $\left|\theta_{4}\right| \leqslant \theta_{3}$.

When $\theta_{i}=\frac{1}{2}(i=1,2,3)$ and $\theta_{4}=-\frac{1}{2}$, the above scheme becomes the $\mathrm{C}-\mathrm{N}$ scheme for BSDE. In 27 the second-order convergence rate of the above scheme was theoretically proved with the parameters $\theta_{i} \in[0,1](i=1,2), \theta_{3} \in(0,1]$, and $\theta_{4} \in[-1,1]$ constrained by $\left|\theta_{4}\right|<\theta_{3}$.

3. By introducing the Gaussian process

$$
\Delta \tilde{W}_{n}=4 \frac{W_{t_{n+1}}-W_{t_{n}}}{\Delta t_{n}}-6 \frac{\int_{t_{n}}^{t_{n+1}}\left(s-t_{n}\right) d W_{s}}{\left(\Delta t_{n}\right)^{2}}
$$

the authors in [5] proposed the following scheme for solving BSDE.

$$
Z^{n}=\mathbb{E}_{t_{n}}\left[\left(Y^{n+1}+\Delta t_{n} f^{n+1}\right) \Delta \tilde{W}_{n}\right], \quad Y_{n}=\mathbb{E}_{t_{n}}\left[Y_{n+1}\right]+\frac{\Delta t_{n}}{2}\left(f^{n}+\mathbb{E}_{t_{n}}\left[f^{n+1}\right]\right),
$$

where $f^{n}=f\left(X^{n}, Y^{n}, Z^{n}\right)$. The authors in 5 only obtained the second-order convergence rate of the above scheme for $X^{n}=X_{t_{n}}$, i.e., the forward SDE was not discretized. Note that the introduced stochastic process $\Delta \tilde{W}_{n}$ in the scheme will cause computation expensive for solving BSDE, and further it is too complex to use the scheme to solve FBSDEs. 


\section{Error estimates of the C-N scheme}

\subsection{Assumptions on approximations of $X_{t}$}

It is obvious that the accuracy of Scheme 3.1 depends on the accuracy of (2.4) for solving the forward $\mathrm{SDE} X_{t}$ in (1.1). In this subsection, to investigate the effect of approximation of forward SDE on the approximation solutions $\left(Y^{n}, Z^{n}\right)$ in the Crank-Nicolson scheme, the following assumptions are made.

Assumption 4.1. Suppose that $X_{0}$ is $\mathcal{F}_{0}$-measurable with $\mathbb{E}\left[\left|X_{0}\right|^{2}\right]<\infty$, and that $b$ and $\sigma$ are $L^{2}$ measurable in $(t, x) \in[0, T] \times \mathbb{R}^{d}$, are linear growth bounded and uniformly Lipschitz continuous, i.e., there exist positive constants $K$ and $L$ such that

$$
\begin{array}{ll}
|b(t, x)|^{2} \leqslant K\left(1+|x|^{2}\right), & |\sigma(t, x)|^{2} \leqslant K\left(1+|x|^{2}\right), \\
|b(t, x)-b(t, y)| \leqslant L|x-y|, & |\sigma(t, x)-\sigma(t, y)| \leqslant L|x-y| .
\end{array}
$$

Assumption 4.2. There is a constant $K^{\prime}>0$ such that the coefficient matrix $\sigma$ satisfies the uniformly elliptic condition

$$
\sigma(t, x) \sigma(t, x)^{\top} \geqslant \frac{1}{K^{\prime}} I_{d \times d}, \quad \forall(t, x) \in[0, T] \times \mathbb{R}^{d} .
$$

Under the Assumption 4.1, if $\mathbb{E}\left[\left|X_{0}\right|^{2 m}\right]<\infty$ for some integer $m \geqslant 1$, the solution of SDE in (2.1) has the estimate

$$
\mathbb{E}\left[\left|X_{s}^{t_{n}, X^{n}}\right|^{2 m}\right] \leqslant\left(1+\left|X^{n}\right|^{2 m}\right) e^{C\left(s-t_{n}\right)},
$$

for any $s \in\left[t_{n}, T\right]$, where $C$ is a positive constant depending only on the constants $K, L$ and $m[13$.

In fact, the weak order-2 Itô-Taylor schemes (2.4) for solving SDE in (1.1) have the following approximation properties and the stability property (see Proposition 5.11.1 in [13] and Assumption 4.2 in $[32$ ).

Assumption 4.3. The approximation solution $X^{n}(n=0,1, \ldots, N-1)$ has the approximation properties

$$
\begin{aligned}
& \left|\mathbb{E}_{t_{n}}^{X^{n}}\left[g\left(X^{n+1}\right)-g\left(X^{n}\right)\right]\right| \leqslant C\left(1+\left|X^{n}\right|^{2 r_{1}}\right) \Delta, \\
& \left|\mathbb{E}_{t_{n}}^{X^{n}}\left[g\left(X_{t_{n+1}}^{t_{n}, X^{n}}\right)-g\left(X^{n+1}\right)\right]\right| \leqslant C\left(1+\left|X^{n}\right|^{2 r_{2}}\right) \Delta^{3}, \\
& \left|\mathbb{E}_{t_{n}}^{X^{n}}\left[\left(g\left(X_{t_{n}+1}^{t_{n}, X^{n}}\right)-g\left(X^{n+1}\right)\right) \Delta W_{n}^{\top}\right]\right| \leqslant C\left(1+\left|X^{n}\right|^{2 r_{3}}\right) \Delta^{3},
\end{aligned}
$$

and the stable estimate property

$$
\max _{0 \leqslant n \leqslant N} \mathbb{E}\left[\left|X^{n}\right|^{r}\right] \leqslant C\left(1+\mathbb{E}\left[\left|X_{0}\right|^{r}\right]\right),
$$

where $r_{i}(i=1,2,3)$ and $r$ are positive integers, and $C$ is a positive constant depending on $g \in C_{b}^{2 \beta+2}$.

The proposition below for the approximation (2.4) of $X_{t}$ holds as well.

Proposition 4.1. Suppose the functions $b, \sigma \in C_{b}^{1,2}$. For $n=0,1, \ldots, N-1$, we have the estimates

$$
\begin{gathered}
\sum_{\alpha \in \Gamma_{2} \backslash\{v\}} \mathbb{E}_{t_{n}}^{X^{n}}\left[\left|g_{\alpha}\left(t_{n+1}, X^{n+1}\right) I_{\alpha, n+1}\right|^{2}\right] \leqslant C \Delta, \\
\mathbb{E}_{t_{n}}^{X^{n}}\left[\left|\sigma\left(t_{n+1}, X^{n+1}\right)-\sigma\left(t_{n}, X^{n}\right)\right|^{2}\right] \leqslant C \Delta,
\end{gathered}
$$

where $C$ is a positive constant independent of $X^{n}, X^{n+1}$, and the time partition.

\subsection{Error equations}

Let $\left(X_{r}^{t, x}, Y_{r}^{t, x}, Z_{r}^{t, x}\right)_{t \leqslant r \leqslant T}$ be the solution of the FBSDEs (2.1) with the terminal condition $Y_{T}^{t, x}=$ $\varphi\left(X_{T}^{t, x}\right),\left(X^{n+1}, Y^{n}, Z^{n}\right)(0 \leqslant n \leqslant N-1)$ be its approximation solution of Scheme 3.1, and let the truncation errors $R_{y 1}^{n}, R_{y 2}^{n}, R_{z 1}^{n}$ and $R_{z 2}^{n}$ be defined in (3.5) and (3.8) for $0 \leqslant n \leqslant N$, respectively.

For the sake of presentation simplicity, we denote

$$
\begin{aligned}
& e_{Y}^{n}:=Y_{t_{n}}^{t_{n}, X^{n}}-Y^{n}, \quad e_{Z}^{n}:=Z_{t_{n}}^{t_{n}, X^{n}}-Z^{n}, \\
& e_{f}^{n}=f\left(t_{n}, X^{n}, Y_{t_{n}}^{t_{n}, X^{n}}, Z_{t_{n}}^{t_{n}, X^{n}}\right)-f\left(t_{n}, X^{n}, Y^{n}, Z^{n}\right),
\end{aligned}
$$


for $n=N-2, \ldots, 1,0$. Subtracting (3.4) and (3.7) from (3.10c) and (3.10b), respectively, we get

$$
\begin{aligned}
& e_{Y}^{n}=\mathbb{E}_{t_{n}}^{X^{n}}\left[e_{Y}^{n+1}\right]+\frac{1}{2} \Delta e_{f}^{n}+\frac{1}{2} \Delta \mathbb{E}_{t_{n}}^{X^{n}}\left[e_{f}^{n+1}\right]+\sum_{j=1}^{2} R_{y j}^{n}, \\
& \Delta e_{Z}^{n}=-\Delta \mathbb{E}_{t_{n}}^{X^{n}}\left[e_{Z}^{n+1}\right]+2 \mathbb{E}_{t_{n}}^{X^{n}}\left[e_{Y}^{n+1} \Delta W_{n}^{\top}\right]+\Delta \mathbb{E}_{t_{n}}^{X^{n}}\left[e_{f}^{n+1} \Delta W_{n}^{\top}\right]+2 \sum_{j=1}^{2} R_{z j}^{n} .
\end{aligned}
$$

Let $e_{\nabla Y}^{n}:=\nabla_{x^{n}} Y_{t_{n}}^{t_{n}, X^{n}}-\nabla_{x^{n}} Y^{n}$ and $e_{\nabla Z}^{n}:=\nabla_{x^{n}} Z_{t_{n}}^{t_{n}, X^{n}}-\nabla_{x^{n}} Z^{n}$. Taking variations w.r.t. $X^{n}$ on both sides of equations (4.8) and (4.9) gives us the following two equations:

$$
e_{\nabla Y}^{n}=\mathbb{E}_{t_{n}}^{X^{n}}\left[e_{\nabla Y}^{n+1} \nabla_{x^{n}} X^{n+1}\right]+\frac{1}{2} \Delta e_{\nabla f}^{n}+\frac{1}{2} \Delta \mathbb{E}_{t_{n}}^{X^{n}}\left[e_{\nabla f}^{n+1} \nabla_{x^{n}} X^{n+1}\right]+\sum_{j=1}^{2} \nabla_{x^{n}} R_{y j}^{n}
$$

and

$$
\begin{aligned}
\Delta e_{\nabla Z}^{n}= & -\Delta \mathbb{E}_{t_{n}}^{X^{n}}\left[e_{\nabla Z}^{n+1} \nabla_{x^{n}} X^{n+1}\right]+2 \mathbb{E}_{t_{n}}^{X^{n}}\left[\Delta W_{n} e_{\nabla Y}^{n+1} \nabla_{x^{n}} X^{n+1}\right] \\
& +\Delta \mathbb{E}_{t_{n}}^{X^{n}}\left[\Delta W_{n} e_{\nabla f}^{n+1} \nabla_{x^{n}} X^{n+1}\right]+2 \sum_{j=1}^{2} \nabla_{x^{n}} R_{z j}^{n},
\end{aligned}
$$

where $\Delta W_{n}=\left(\Delta W_{n}^{1}, \ldots, \Delta W_{n}^{d}\right)^{\top}, e_{\nabla Y}^{n+2}:=\left(e_{\nabla Y}^{1, n+2}, \ldots, e_{\nabla Y}^{d, n+2}\right)=\left(\nabla_{x_{1}^{n}} e_{Y}^{n+2}, \ldots, \nabla_{x_{d}^{n}} e_{Y}^{n+2}\right)$, and

$$
\begin{aligned}
e_{\nabla f}^{n}= & f_{X}^{t_{n}, X^{n}}-f_{X}^{n}+\left(f_{Y}^{t_{n}, X^{n}}-f_{Y}^{n}\right) \nabla_{x^{n}} Y_{t_{n}}^{t_{n}, X^{n}}+f_{Y}^{n} e_{\nabla Y}^{n} \\
& +\left(f_{Z}^{t_{n}, X^{n}}-f_{Z}^{n}\right) \nabla_{x^{n}} Z_{t_{n}}^{t_{n}, X^{n}}+f_{Z}^{n} e_{\nabla Z}^{n} .
\end{aligned}
$$

\subsection{Main error estimate results}

Now we state our main error estimate results in Theorems 4.1 and 4.2 below.

Theorem 4.1. For the weak order-2 Itô-Taylor approximation $X^{n+1}$ satisfying (2.4), if $b, \sigma \in C_{b}^{1,3}$ and $f \in C_{b}^{1,2,2,2}$, then under Hypotheses 4.1, for $0 \leqslant n \leqslant N-2$, it holds that

$$
\begin{aligned}
& \mathbb{E}\left[\left|e_{Y}^{n}\right|^{2}+\left|e_{Z}^{n}\right|^{2}+\left|e_{\nabla Y}^{n}\right|^{2}+\Delta\left|e_{\nabla Z}^{n}\right|^{2}\right] \\
& \leqslant C \mathbb{E}\left[\left|e_{Y}^{N-1}\right|^{2}+\left|e_{Z}^{N-1}\right|^{2}+\left|e_{\nabla Y}^{N-1}\right|^{2}+\Delta\left|e_{\nabla Z}^{N-1}\right|^{2}\right] \\
&+C \sum_{i=n}^{N-2} \sum_{j=1}^{2} \mathbb{E}\left[\frac{1}{\Delta^{3}}\left(\left|\mathbb{E}_{t_{i}}^{X^{i}}\left[R_{y j}^{i+1} \Delta W_{i}^{\top}\right]\right|^{2}+\left|R_{z j}^{i}-\mathbb{E}_{t_{i}}^{X^{i}}\left[R_{z j}^{i+1}\right]\right|^{2}\right)\right. \\
&+\frac{1}{\Delta}\left(\left|\mathbb{E}_{t_{i}}^{X^{i}}\left[R_{y j}^{i+1}\right]\right|^{2}+\left|\mathbb{E}_{t_{i}}^{X^{i}}\left[\nabla_{x^{i}} R_{y j}^{i+1}\right]\right|^{2}+\left|\mathbb{E}_{t_{i}}^{X^{i}}\left[\nabla_{x^{i}} R_{z j}^{i+1}\right]\right|^{2}\right. \\
&+\left|\nabla_{x^{i}} \mathbb{E}_{t_{i}}^{X^{i}}\left[R_{y j}^{i+1} \Delta W_{i}^{\top}\right]\right|^{2}+\left|R_{y j}^{i}\right|^{2}+\left|\nabla_{x^{i}} R_{y j}^{i}\right|^{2}+\left|\nabla_{x^{i}} R_{z j}^{i}\right|^{2} \\
&\left.\left.+\mathbb{E}_{t_{i}}^{X^{i}}\left[\left|R_{z j}^{i+1}\right|^{2}\right]+\mathbb{E}_{t_{i}}^{X^{i}}\left[\left|\nabla_{x^{i+1}} R_{z j}^{i+1}\right|^{2}\right]\right)\right]
\end{aligned}
$$

where $C$ is a generic positive constant depending on $d, T, K^{\prime}$, and upper bounds of derivatives of $b, \sigma$ and $f$.

Theorem 4.2. Suppose $b, \sigma \in C_{b}^{3,6}, f \in C_{b}^{3,6,6,6}$, and $\varphi \in C_{b}^{7+\alpha}$ for some $\alpha \in(0,1)$. Then for the weak order-2 Itô-Taylor approximation solution $X^{n+1}, 0 \leqslant n \leqslant N-2$, under Assumptions 4.14.3, it holds that

$$
\max _{0 \leqslant n \leqslant N} \mathbb{E}\left[\left|e_{Y}^{n}\right|^{2}+\left|e_{Z}^{n}\right|^{2}+\left|e_{\nabla Y}^{n}\right|^{2}+\Delta\left|e_{\nabla Z}^{n}\right|^{2}\right] \leqslant C \Delta^{4},
$$

where $C$ is a generic positive constant depending on $d, T, K^{\prime}, K, L$, the initial value of $X_{t}$ in (1.1), and upper bounds of derivatives of $b, \sigma, f$ and $\varphi$.

Remark 4.1. Scheme 3.1 is stable, which is implied by Theorem 4.1, and its solution continuously depends on terminal condition. That is, for any given positive number $\varepsilon$, there exits a positive number $\delta$, for different terminal conditions $\left(Y_{1}^{N}, Z_{1}^{N}\right)$ and $\left(Y_{2}^{N}, Z_{2}^{N}\right)$, if $\mathbb{E}\left[\left|Y_{1}^{N}-Y_{2}^{N}\right|^{2}\right]<\delta$ and $\mathbb{E}\left[\left|Z_{1}^{N}-Z_{2}^{N}\right|^{2}\right]<\delta$, then for $0 \leqslant n \leqslant N-1$, we have

$$
\mathbb{E}\left[\left|Y_{1}^{n}-Y_{2}^{n}\right|^{2}+\left|Z_{1}^{n}-Z_{2}^{n}\right|^{2}\right]<\varepsilon .
$$




\section{Proofs of the main results}

In this section, we will give rigorous proofs of Theorems 4.1 and 4.2 . In the sequel, we will use $V a r^{n}(G)$ to denote the conditional variance of random variable $G$, i.e., $\operatorname{Var}^{n}(G)=\mathbb{E}_{t_{n}}^{X^{n}}\left[|G|^{2}\right]-\left|\mathbb{E}_{t_{n}}^{X^{n}}[G]\right|^{2}$.

Before giving the proof of Theorem 4.1, we introduce the following useful lemma.

Lemma 5.0.1. For the weak order-2 Itô-Taylor approximation $X^{n+1}$ satisfying (2.4), let $b, \sigma \in C_{b}^{1,3}$ and $f \in C_{b}^{1,2,2,2}$. Then for $0 \leqslant n \leqslant N-2$, it holds that

$$
\begin{aligned}
& \Delta\left(\left|e_{Y}^{n}\right|^{2}+\left|e_{Z}^{n}\right|^{2}+\left|e_{\nabla Y}^{n}\right|^{2}+\left|e_{\nabla Z}^{n}\right|^{2}\right) \\
\leqslant & C \Delta \mathbb{E}_{t_{n}}^{X^{n}}\left[\left|e_{Y}^{n+1}\right|^{2}+\left|e_{Z}^{n+1}\right|^{2}+\left|e_{\nabla Y}^{n+1}\right|^{2}+\left|e_{\nabla Z}^{n+1}\right|^{2}\right]+C \operatorname{Var}^{n}\left(e_{Y}^{n+1}\right)+C \operatorname{Var}^{n}\left(e_{\nabla Y}^{n+1}\right) \\
+ & C \sum_{j=1}^{2}\left(\Delta\left|R_{y j}^{n}\right|^{2}+\Delta\left|\nabla_{x^{n}} R_{y j}^{n}\right|^{2}+\frac{1}{\Delta}\left|R_{z j}^{n}\right|^{2}+\frac{1}{\Delta}\left|\nabla_{x^{n}} R_{z j}^{n}\right|^{2}\right),
\end{aligned}
$$

where $C$ is a positive generic constant depending only on $d$, and upper bounds of derivatives of $b, \sigma, f$ and $\varphi$.

Proof. By (4.8) and the Lipschitz continuity of function $f$, we easily deduce

$$
\left|e_{Y}^{n}\right| \leqslant\left(1+\frac{L^{\prime}}{2} \Delta\right) \mathbb{E}_{t_{n}}^{X^{n}}\left[\left|e_{Y}^{n+1}\right|\right]+\frac{L^{\prime}}{2} \Delta\left(\left|e_{Y}^{n}\right|+\left|e_{Z}^{n}\right|+\mathbb{E}_{t_{n}}^{X^{n}}\left[\left|e_{Z}^{n+1}\right|\right]\right)+\sum_{j=1}^{2}\left|R_{y j}^{n}\right|
$$

where $L^{\prime}$ is the Lipschitz constant. Then taking square on both sides of the above inequality and using the inequality $\left(\sum_{i=1}^{m} a_{i}\right)^{2} \leqslant m \sum_{i=1}^{m} a_{i}^{2}$, we deduce

$$
\left|e_{Y}^{n}\right|^{2} \leqslant C \mathbb{E}_{t_{n}}^{X^{n}}\left[\left|e_{Y}^{n+1}\right|^{2}\right]+C \Delta^{2}\left(\left|e_{Y}^{n}\right|^{2}+\left|e_{Z}^{n}\right|^{2}+\mathbb{E}_{t_{n}}^{X^{n}}\left[\left|e_{Z}^{n+1}\right|^{2}\right]\right)+C \sum_{j=1}^{2}\left|R_{y j}^{n}\right|^{2}
$$

Similarly, from the error equations (4.9) w.11) we obtain

$$
\begin{gathered}
\Delta\left|e_{Z}^{n}\right|^{2} \leqslant \\
C \Delta \mathbb{E}_{t_{n}}^{X^{n}}\left[\left|e_{Z}^{n+1}\right|^{2}\right]+C \operatorname{Var}^{n}\left(e_{Y}^{n+1}\right) \\
+C \Delta^{2} \mathbb{E}_{t_{n}}^{X^{n}}\left[\left|e_{Y}^{n+1}\right|^{2}+\left|e_{Z}^{n+1}\right|^{2}\right]+\frac{C}{\Delta} \sum_{j=1}^{2}\left|R_{z j}^{n}\right|^{2}, \\
\left|e_{\nabla Y}^{n}\right|^{2} \leqslant C \mathbb{E}_{t_{n}}^{X^{n}}\left[\left|e_{\nabla Y}^{n+1}\right|^{2}\right]+C \Delta^{2}\left(\left|e_{\nabla Y}^{n}\right|^{2}+\left|e_{\nabla Z}^{n}\right|^{2}+\left|e_{Y}^{n}\right|^{2}+\left|e_{Z}^{n}\right|^{2}\right) \\
+C \Delta^{2} \mathbb{E}_{t_{n}}^{X^{n}}\left[\left|e_{\nabla Y}^{n+1}\right|^{2}+\left|e_{\nabla Z}^{n+1}\right|^{2}+\left|e_{Y}^{n+1}\right|^{2}+\left|e_{Z}^{n+1}\right|^{2}\right]+C \sum_{j=1}^{2}\left|\nabla_{x^{n}} R_{y j}^{n}\right|^{2},
\end{gathered}
$$

and

$$
\begin{aligned}
\Delta\left|e_{\nabla Z}^{n}\right|^{2} \leqslant & C \Delta \mathbb{E}_{t_{n}}^{X^{n}}\left[\left|e_{\nabla Z}^{n+1}\right|^{2}\right]+C \operatorname{Var}^{n}\left(e_{\nabla Y}^{n+1}\right) \\
& +C \Delta \mathbb{E}_{t_{n}}^{X^{n}}\left[\left|e_{Y}^{n+1}\right|^{2}+\left|e_{Z}^{n+1}\right|^{2}+\left|e_{\nabla Y}^{n+1}\right|^{2}+\left|e_{\nabla Z}^{n+1}\right|^{2}\right]+\frac{C}{\Delta} \sum_{j=1}^{2}\left|\nabla_{x^{n}} R_{z j}^{n}\right|^{2} .
\end{aligned}
$$

Now combining the above four inequalities yields

$$
\begin{aligned}
& \Delta\left(\left|e_{Y}^{n}\right|^{2}+\left|e_{Z}^{n}\right|^{2}+\left|e_{\nabla Y}^{n}\right|^{2}+\left|e_{\nabla Z}^{n}\right|^{2}\right) \\
\leqslant & C \Delta \mathbb{E}_{t_{n}}^{X^{n}}\left[\left|e_{Y}^{n+1}\right|^{2}+\left|e_{Z}^{n+1}\right|^{2}+\left|e_{\nabla Y}^{n+1}\right|^{2}+\left|e_{\nabla Z}^{n+1}\right|^{2}\right]+C \operatorname{Var}^{n}\left(e_{Y}^{n+1}\right)+C \operatorname{Var}^{n}\left(e_{\nabla Y}^{n+1}\right) \\
+ & C \sum_{j=1}^{2}\left(\Delta\left|R_{y j}^{n}\right|^{2}+\Delta\left|\nabla_{x^{n}} R_{y j}^{n}\right|^{2}+\frac{1}{\Delta}\left(\left|R_{z j}^{n}\right|^{2}+\left|\nabla_{x^{n}} R_{z j}^{n}\right|^{2}\right)\right) .
\end{aligned}
$$

The proof is completed. 


\subsection{Proof of Theorem 4.1}

Now, we give the proof of Theorem 4.1 which is divided into five steps. In each step of the proof except the last step, we deduce an estimate for $e_{Y}^{n}, e_{Z}^{n}, e_{\nabla Y}^{n}$, and $e_{\nabla Z}^{n}$ successively.

Proof. (1) The estimate of $e_{Y}^{n}$.

By (4.8) we have

$$
e_{Y}^{n+1}=\mathbb{E}_{t_{n+1}}^{X^{n+1}}\left[e_{Y}^{n+2}\right]+\frac{1}{2} \Delta\left(e_{f}^{n+1}+\mathbb{E}_{t_{n+1}}^{X^{n+1}}\left[e_{f}^{n+2}\right]\right)+\sum_{j=1}^{2} R_{y j}^{n+1}
$$

Inserting the $e_{Y}^{n+1}$ in (5.2) into (4.8) gives

$$
e_{Y}^{n}=\mathbb{E}_{t_{n}}^{X^{n}}\left[\mathbb{E}_{t_{n+1}}^{X^{n+1}}\left[e_{Y}^{n+2}\right]\right]+\frac{1}{2} \Delta e_{f}^{n}+\Delta \mathbb{E}_{t_{n}}^{X^{n}}\left[e_{f}^{n+1}+\frac{1}{2} e_{f}^{n+2}\right]+\sum_{j=1}^{2}\left(R_{y j}^{n}+\mathbb{E}_{t_{n}}^{X^{n}}\left[R_{y j}^{n+1}\right]\right)
$$

Then taking square on both sides of the above inequality and using Young's inequality $(a+b)^{2} \leqslant$ $\left(1+\gamma_{1} \Delta\right) a^{2}+\left(1+\frac{1}{\gamma_{1} \Delta}\right) b^{2}$ (for any $\left.\gamma_{1}>0\right)$ yield

$$
\begin{aligned}
\left|e_{Y}^{n}\right|^{2} \leqslant & \left(1+\gamma_{1} \Delta\right) \mathbb{E}_{t_{n}}^{X^{n}}\left[\left|\mathbb{E}_{t_{n+1}}^{X^{n+1}}\left[e_{Y}^{n+2}\right]\right|^{2}\right]+\frac{C}{\gamma_{1}}\left(1+\gamma_{1} \Delta\right)\left\{\Delta\left(\left|e_{Y}^{n}\right|^{2}+\left|e_{Z}^{n}\right|^{2}\right)\right. \\
& \left.+\Delta \mathbb{E}_{t_{n}}^{X^{n}}\left[\left|e_{Y}^{n+1}\right|^{2}+\left|e_{Z}^{n+1}\right|^{2}+\left|e_{Y}^{n+2}\right|^{2}+\left|e_{Z}^{n+2}\right|^{2}\right]+\sum_{j=1}^{2} \frac{\left|R_{y j}^{n}\right|^{2}+\left|\mathbb{E}_{t_{n}}^{X^{n}}\left[R_{y j}^{n+1}\right]\right|^{2}}{\Delta}\right\} .
\end{aligned}
$$

(2) The estimate of $e_{Z}^{n}$.

Replacing the $n$ in (4.9) by $n+1$, we deduce

$$
-\mathbb{E}_{t_{n}}^{X^{n}}\left[e_{Z}^{n+1}\right]=\mathbb{E}_{t_{n}}^{X^{n}}\left[e_{Z}^{n+2}\right]-\frac{2}{\Delta} \mathbb{E}_{t_{n}}^{X^{n}}\left[e_{Y}^{n+2} \Delta W_{n+1}^{\top}\right]-\mathbb{E}_{t_{n}}^{X^{n}}\left[e_{f}^{n+2} \Delta W_{n+1}^{\top}\right]-\frac{2}{\Delta} \sum_{j=1}^{2} \mathbb{E}_{t_{n}}^{X^{n}}\left[R_{z j}^{n+1}\right]
$$

Inserting $e_{Y}^{n+1}$ in (5.2) and $-\mathbb{E}_{t_{n}}^{X^{n}}\left[e_{Z}^{n+1}\right]$ in (5.5) into (4.9), we deduce

$$
\begin{aligned}
e_{Z}^{n}= & \mathbb{E}_{t_{n}}^{X^{n}}\left[e_{Z}^{n+2}\right]+\frac{2}{\Delta} \mathbb{E}_{t_{n}}^{X^{n}}\left[e_{Y}^{n+2}\left(\Delta W_{n}^{\top}-\Delta W_{n+1}^{\top}\right)\right] \\
& +\mathbb{E}_{t_{n}}^{X^{n}}\left[e_{f}^{n+2} \Delta W_{n}^{\top}\right]-\mathbb{E}_{t_{n}}^{X^{n}}\left[e_{f}^{n+2} \Delta W_{n+1}^{\top}\right]+2 \mathbb{E}_{t_{n}}^{X^{n}}\left[e_{f}^{n+1} \Delta W_{n}^{\top}\right] \\
& +\sum_{j=1}^{2} \frac{2}{\Delta}\left(\mathbb{E}_{t_{n}}^{X^{n}}\left[R_{y j}^{n+1} \Delta W_{n}^{\top}\right]+R_{z j}^{n}-\mathbb{E}_{t_{n}}^{X^{n}}\left[R_{z j}^{n+1}\right]\right) .
\end{aligned}
$$

By the Malliavin integration-by-parts formula (2.9) and the chain rule (2.10), we have

$$
\mathbb{E}_{t_{n}}^{X^{n}}\left[e_{Y}^{n+2}\left(\Delta W_{n}^{\top}-\Delta W_{n+1}^{\top}\right)\right]=\mathbb{E}_{t_{n}}^{X^{n}}\left[e_{\nabla Y}^{n+2}\left(\int_{t_{n}}^{t_{n+1}} D_{t} X^{n+2} d t-\int_{t_{n+1}}^{t_{n+2}} D_{t} X^{n+2} d t\right)\right],
$$

where $D_{t} X^{n+2}=\left[D_{1, t} X^{n+2}, \ldots, D_{d, t} X^{n+2}\right]_{d \times d}$ is a $d \times d$ square matrix with

$$
D_{j, t} X^{n+2}=\left(D_{j, t} X_{1}^{n+2}, \ldots, D_{j, t} X_{d}^{n+2}\right)^{\top} \quad \text { for } \quad 1 \leqslant j \leqslant d
$$

The weak order-2 Itô-Taylor approximation solution $X^{n+2}$ can be represented as

$$
\begin{aligned}
X^{n+2}= & X^{n+1}+b\left(t_{n+1}, X^{n+1}\right) \Delta+\sigma\left(t_{n+1}, X^{n+1}\right) \Delta W_{n+1} \\
& +\sum_{\alpha \in \mathcal{A}_{2}} g_{\alpha}\left(t_{n+1}, X^{n+1}\right) I_{\alpha, n+1} .
\end{aligned}
$$


Taking the Malliavin derivative $D_{t}$ to both sides of the above equation yields

$$
\int_{t_{n+1}}^{t_{n+2}} D_{t} X^{n+2} d t=\sigma\left(t_{n+1}, X^{n+1}\right) \Delta+\sum_{\alpha \in \mathcal{A}_{2}} g_{\alpha}\left(t_{n+1}, X^{n+1}\right) \int_{t_{n+1}}^{t_{n+2}} D_{t} I_{\alpha, n+1} d t
$$

and

$$
\begin{aligned}
\int_{t_{n}}^{t_{n+1}} D_{t} X^{n+2} d t= & \int_{t_{n}}^{t_{n+1}} D_{t} X^{n+1} d t+\sum_{\alpha \in \Gamma_{2} \backslash\{v\}} \int_{t_{n}}^{t_{n+1}} D_{t}\left\{g_{\alpha}\left(t_{n+1}, X^{n+1}\right) I_{\alpha, n+1}\right\} d t \\
= & \sigma\left(t_{n}, X^{n}\right) \Delta+\sum_{\alpha \in \mathcal{A}_{2}} g_{\alpha}\left(t_{n}, X^{n}\right) \int_{t_{n}}^{t_{n+1}} D_{t} I_{\alpha, n} d t \\
& +\sum_{\alpha \in \Gamma_{2} \backslash\{v\}} \int_{t_{n}}^{t_{n+1}} D_{t}\left\{g_{\alpha}\left(t_{n+1}, X^{n+1}\right) I_{\alpha, n+1}\right\} d t .
\end{aligned}
$$

By (5.7), (5.8) and (5.9), we deduce

$$
\begin{aligned}
& \mathbb{E}_{t_{n}}^{X^{n}}\left[e_{Y}^{n+2}\left(\Delta W_{n}^{\top}-\Delta W_{n+1}^{\top}\right)\right] \\
= & \mathbb{E}_{t_{n}}^{X^{n}}\left[e_{\nabla Y}^{n+2}\left(\sigma\left(t_{n}, X^{n}\right)-\sigma\left(t_{n+1}, X^{n+1}\right)\right)\right] \Delta+\sum_{\alpha \in \mathcal{A}_{2}} \mathbb{E}_{t_{n}}^{X^{n}}\left[e_{\nabla Y}^{n+2} g_{\alpha}\left(t_{n}, X^{n}\right) \int_{t_{n}}^{t_{n+1}} D_{t} I_{\alpha, n} d t\right] \\
& -\sum_{\alpha \in \mathcal{A}_{2}} \mathbb{E}_{t_{n}}^{X^{n}}\left[e_{\nabla Y}^{n+2} g_{\alpha}\left(t_{n+1}, X^{n+1}\right) \int_{t_{n+1}}^{t_{n+2}} D_{t} I_{\alpha, n+1} d t\right] \\
& +\sum_{\alpha \in \Gamma_{2} \backslash\{v\}} \int_{t_{n}}^{t_{n+1}} \mathbb{E}_{t_{n}}^{X^{n}}\left[e_{\nabla Y}^{n+2} D_{t}\left\{g_{\alpha}\left(t_{n+1}, X^{n+1}\right) I_{\alpha, n+1}\right\}\right] d t .
\end{aligned}
$$

Also,

$$
\begin{aligned}
& \mathbb{E}_{t_{n}}^{X^{n}}\left[e_{f}^{n+1} \Delta W_{n}^{\top}\right]=\int_{t_{n}}^{t_{n+1}} \mathbb{E}_{t_{n}}^{X^{n}}\left[D_{t} e_{f}^{n+1}\right] d t=\int_{t_{n}}^{t_{n+1}} \mathbb{E}_{t_{n}}^{X^{n}}\left[e_{\nabla f}^{n+1} D_{t} X^{n+1}\right] d t, \\
& \mathbb{E}_{t_{n}}^{X^{n}}\left[e_{f}^{n+2} \Delta W_{n}^{\top}\right]=\int_{t_{n}}^{t_{n+1}} \mathbb{E}_{t_{n}}^{X^{n}}\left[e_{\nabla f}^{n+2} D_{t} X^{n+2}\right] d t, \\
& \mathbb{E}_{t_{n}}^{X^{n}}\left[e_{f}^{n+2} \Delta W_{n+1}^{\top}\right]=\int_{t_{n+1}}^{t_{n+2}} \mathbb{E}_{t_{n}}^{X^{n}}\left[e_{\nabla f}^{n+2} D_{t} X^{n+2}\right] d t .
\end{aligned}
$$

Under the assumption $b, \sigma \in C_{b}^{1,2}$ and Proposition 4.1, using the equalities in (5.10), (5.11) and the Hölder inequality, we have

$$
\begin{aligned}
& \left|\mathbb{E}_{t_{n}}^{X^{n}}\left[e_{Y}^{n+2}\left(\Delta W_{n}^{\top}-\Delta W_{n+1}^{\top}\right)\right]\right|^{2} \leqslant C \Delta^{3} \mathbb{E}_{t_{n}}^{X^{n}}\left[\left|e_{\nabla Y}^{n+2}\right|^{2}\right], \\
& \left|\mathbb{E}_{t_{n}}^{X^{n}}\left[e_{f}^{n+1} \Delta W_{n}^{\top}\right]\right|^{2} \leqslant C \Delta^{2} \mathbb{E}_{t_{n}}^{X^{n}}\left[\left|e_{Y}^{n+1}\right|^{2}+\left|e_{Z}^{n+1}\right|^{2}+\left|e_{\nabla Y}^{n+1}\right|^{2}+\left|e_{\nabla Z}^{n+1}\right|^{2}\right], \\
& \left|\mathbb{E}_{t_{n}}^{X^{n}}\left[e_{f}^{n+2} \Delta W_{n}^{\top}\right]\right|^{2} \leqslant C \Delta^{2} \mathbb{E}_{t_{n}}^{X^{n}}\left[\left|e_{Y}^{n+2}\right|^{2}+\left|e_{Z}^{n+2}\right|^{2}+\left|e_{\nabla Y}^{n+2}\right|^{2}+\left|e_{\nabla Z}^{n+2}\right|^{2}\right], \\
& \left|\mathbb{E}_{t_{n}}^{X^{n}}\left[e_{f}^{n+2} \Delta W_{n+1}^{\top}\right]\right|^{2} \leqslant C \Delta^{2} \mathbb{E}_{t_{n}}^{X^{n}}\left[\left|e_{Y}^{n+2}\right|^{2}+\left|e_{Z}^{n+2}\right|^{2}+\left|e_{\nabla Y}^{n+2}\right|^{2}+\left|e_{\nabla Z}^{n+2}\right|^{2}\right] .
\end{aligned}
$$

Similarly, by taking square on both sides of the equation (5.6), and using the inequalities in (5.12) and the Young's inequality again, we obtain

$$
\begin{aligned}
\left|e_{Z}^{n}\right|^{2} \leqslant & \left(1+\gamma_{2} \Delta\right)\left|\mathbb{E}_{t_{n}}^{X^{n}}\left[e_{Z}^{n+2}\right]\right|^{2}+\frac{C}{\gamma_{2}}\left(1+\gamma_{2} \Delta\right)\left\{\Delta \mathbb { E } _ { t _ { n } } ^ { X _ { n } ^ { n } } \left[\left|e_{Y}^{n+1}\right|^{2}+\left|e_{Z}^{n+1}\right|^{2}\right.\right. \\
& \left.+\left|e_{\nabla Y}^{n+1}\right|^{2}+\left|e_{\nabla Z}^{n+1}\right|^{2}+\left|e_{Y}^{n+2}\right|^{2}+\left|e_{Z}^{n+2}\right|^{2}+\left|e_{\nabla Y}^{n+2}\right|^{2}+\left|e_{\nabla Z}^{n+2}\right|^{2}\right] \\
& \left.+\sum_{j=1}^{2} \frac{\left|\mathbb{E}_{t_{n}}^{X^{n}}\left[R_{y j}^{n+1} \Delta W_{n}^{\top}\right]\right|^{2}+\left|R_{z j}^{n}-\mathbb{E}_{t_{n}}^{X^{n}}\left[R_{z j}^{n+1}\right]\right|^{2}}{\Delta^{3}}\right\} .
\end{aligned}
$$

(3) The estimate of $e_{\nabla Y}^{n}$. 
Taking variation on both sides of the equation (5.3) gives

$$
\begin{aligned}
e_{\nabla Y}^{n}= & \mathbb{E}_{t_{n}}^{X^{n}}\left[e_{\nabla Y}^{n+2} \nabla_{x^{n}} X^{n+2}\right]+\frac{1}{2} \Delta e_{\nabla f}^{n}+\Delta \mathbb{E}_{t_{n}}^{X^{n}}\left[e_{\nabla f}^{n+1} \nabla_{x^{n}} X^{n+1}\right] \\
& +\frac{1}{2} \Delta \mathbb{E}_{t_{n}}^{X^{n}}\left[e_{\nabla f}^{n+2} \nabla_{x^{n}} X^{n+2}\right]+\sum_{j=1}^{2}\left(\nabla_{x^{n}} R_{y j}^{n}+\mathbb{E}_{t_{n}}^{X^{n}}\left[\nabla_{x^{n}} R_{y j}^{n+1}\right]\right) .
\end{aligned}
$$

Using the Taylor expansion to $\sigma\left(t_{n+1}, X^{n+1}\right)$, we have

$$
\sigma\left(t_{n+1}, X^{n+1}\right)=\sigma\left(t_{n+1}, X^{n}\right)+\int_{0}^{1} \sigma_{x}\left(t_{n+1}, X^{n}+\lambda\left(X^{n+1}-X^{n}\right)\right)\left(X^{n+1}-X^{n}\right) d \lambda
$$

which combining the equation (5.1) implies

$$
X^{n+2}=X^{n}+\sigma\left(t_{n}, X^{n}\right) \Delta W_{n}+\sigma\left(t_{n+1}, X^{n}\right) \Delta W_{n+1}+\Lambda_{n},
$$

where

$$
\begin{aligned}
\Lambda_{n}= & b\left(t_{n}, X^{n}\right) \Delta+\sum_{\alpha \in \mathcal{A}_{2}} g_{\alpha}\left(t_{n}, X^{n}\right) I_{\alpha, n} \\
& +b\left(t_{n+1}, X^{n+1}\right) \Delta+\sum_{\alpha \in \mathcal{A}_{2}} g_{\alpha}\left(t_{n+1}, X^{n+1}\right) I_{\alpha, n+1} \\
& +\int_{0}^{1} \sigma_{x}\left(t_{n+1}, X^{n}+\lambda\left(X^{n+1}-X^{n}\right)\right)\left(X^{n+1}-X^{n}\right) \Delta W_{n+1} d \lambda .
\end{aligned}
$$

Taking variation on both sides of the equation (5.15) gives

$$
\nabla_{x^{n}} X^{n+2}=I_{d \times d}+\sigma_{x}\left(t_{n}, X^{n}\right) \Delta W_{n}+\sigma_{x}\left(t_{n+1}, X^{n}\right) \Delta W_{n+1}+\nabla_{x^{n}} \Lambda_{n} .
$$

Then,

$$
\begin{aligned}
\mathbb{E}_{t_{n}}^{X^{n}}\left[e_{\nabla Y}^{n+2} \nabla_{x^{n}} X^{n+2}\right]= & \mathbb{E}_{t_{n}}^{X^{n}}\left[e_{\nabla Y}^{n+2}\right]+\sigma_{x}\left(t_{n}, X^{n}\right) \mathbb{E}_{t_{n}}^{X^{n}}\left[e_{\nabla Y}^{n+2} \Delta W_{n}^{\top}\right] \\
& +\sigma_{x}\left(t_{n+1}, X^{n}\right) \mathbb{E}_{t_{n}}^{X^{n}}\left[e_{\nabla Y}^{n+2} \Delta W_{n+1}^{\top}\right]+\mathbb{E}_{t_{n}}^{X^{n}}\left[e_{\nabla Y}^{n+2} \nabla_{x^{n}} \Lambda_{n}\right] .
\end{aligned}
$$

By the Hölder inequality, under the assumption $b, \sigma \in C_{b}^{1,3}$, we get

$$
\begin{aligned}
& \mathbb{E}_{t_{n}}^{X^{n}}\left[\left|\nabla_{X^{n}} \Lambda_{n}\right|^{2}\right] \leqslant C\left(1+\left|X^{n}\right|^{q}\right) \Delta^{2}, \\
& \left|\sigma_{x}\left(t_{n}, X^{n}\right) \mathbb{E}_{t_{n}}^{X^{n}}\left[e_{\nabla Y}^{n+2} \Delta W_{n}^{\top}\right]\right|^{2} \leqslant C \Delta \operatorname{Var}^{n}\left(e_{\nabla Y}^{n+2}\right), \\
& \left|\sigma_{x}\left(t_{n+1}, X^{n}\right) \mathbb{E}_{t_{n}}^{X^{n}}\left[e_{\nabla Y}^{n+2} \Delta W_{n+1}^{\top}\right]\right|^{2} \leqslant C \Delta \operatorname{Var}^{n}\left(e_{\nabla Y}^{n+2}\right) .
\end{aligned}
$$

Taking square on both sides of the equation (5.14) and using Young's inequality $(a+b)^{2} \leqslant\left(1+\gamma_{3} \Delta\right) a^{2}+$ $\left(1+\frac{1}{\gamma_{3} \Delta}\right) b^{2}$ (for any $\left.\gamma_{3}>0\right)$, we obtain

$$
\begin{aligned}
\left|e_{\nabla Y}^{n}\right|^{2} \leqslant & \left(1+\gamma_{3} \Delta\right)\left|\mathbb{E}_{t_{n}}^{X^{n}}\left[e_{\nabla Y}^{n+2}\right]\right|^{2}+\frac{C}{\gamma_{3}}\left(1+\gamma_{3} \Delta\right)\left\{\Delta\left(\left|e_{Y}^{n}\right|^{2}+\left|e_{Z}^{n}\right|^{2}+\left|e_{\nabla Y}^{n}\right|^{2}+\left|e_{\nabla Z}^{n}\right|^{2}\right)\right. \\
& +\operatorname{Var}^{n}\left(e_{\nabla Y}^{n+2}\right)+\Delta \mathbb{E}_{t_{n}}^{X^{n}}\left[\left|e_{Y}^{n+1}\right|^{2}+\left|e_{Z}^{n+1}\right|^{2}+\left|e_{\nabla Y}^{n+1}\right|^{2}+\left|e_{\nabla Z}^{n+1}\right|^{2}+\left|e_{Y}^{n+2}\right|^{2}\right. \\
& \left.\left.+\left|e_{Z}^{n+2}\right|^{2}+\left|e_{\nabla Y}^{n+2}\right|^{2}+\left|e_{\nabla Z}^{n+2}\right|^{2}\right]+\sum_{j=1}^{2} \frac{\left|\nabla_{x^{n}} R_{y j}^{n}\right|^{2}+\left|\mathbb{E}_{t_{n}}^{X^{n}}\left[\nabla_{x^{n}} R_{y j}^{n+1}\right]\right|^{2}}{\Delta}\right\} .
\end{aligned}
$$

(4) The estimate of $e_{\nabla Z}^{n}$.

Taking variation on both sides of the equation (5.6) leads to

$$
\begin{aligned}
\Delta e_{\nabla Z}^{n}= & \Delta \mathbb{E}_{t_{n}}^{X_{n}^{n}}\left[e_{\nabla Z}^{n+2} \nabla_{x^{n}} X^{n+2}\right]-2 \mathbb{E}_{t_{n}}^{X^{n}}\left[\Delta W_{n+1} e_{\nabla Y}^{n+2} \nabla_{x^{n}} X^{n+2}\right] \\
& +2 \mathbb{E}_{t_{n}}^{X^{n}}\left[\Delta W_{n} e_{\nabla Y}^{n+2} \nabla_{x^{n}} X^{n+2}\right]+\Delta \mathbb{E}_{t_{n}}^{X^{n}}\left[\Delta W_{n} e_{\nabla f}^{n+2} \nabla_{x^{n}} X^{n+2}\right] \\
& -\Delta \mathbb{E}_{t_{n}}^{X^{n}}\left[\Delta W_{n+1} e_{\nabla f}^{n+2} \nabla_{x^{n}} X^{n+2}\right]+2 \Delta \mathbb{E}_{t_{n}}^{X^{n}}\left[\Delta W_{n} e_{\nabla f}^{n+1} \nabla_{x^{n}} X^{n+1}\right] \\
& +2 \sum_{j=1}^{2}\left(\mathbb{E}_{t_{n}}^{X^{n}}\left[\Delta W_{n} \nabla_{x^{n}} R_{y j}^{n+1}\right]+\nabla_{x^{n}} R_{z j}^{n}-\mathbb{E}_{t_{n}}^{X^{n}}\left[\nabla_{x^{n}} R_{z j}^{n+1}\right]\right) .
\end{aligned}
$$


By the facts $\nabla_{x^{n}} X^{n+1}=I_{d \times d}+\sum_{\alpha \in \Gamma_{2} \backslash\{v\}} \partial_{x} g_{\alpha}\left(t_{n}, X^{n}\right) I_{\alpha, n}$ and

$$
\nabla_{x^{n}} X^{n+2}=\nabla_{x^{n+1}} X^{n+2} \nabla_{x^{n}} X^{n+1}=\left(I_{d \times d}+\sum_{\alpha \in \Gamma_{2} \backslash\{v\}} \partial_{x} g_{\alpha}\left(t_{n+1}, X^{n+1}\right) I_{\alpha, n+1}\right) \nabla_{x^{n}} X^{n+1},
$$

and using the Hölder inequality, we have the estimate

$$
\begin{aligned}
& \left|\mathbb{E}_{t_{n}}^{X^{n}}\left[\Delta W_{n} e_{\nabla Y}^{n+2} \nabla_{x^{n}} X^{n+2}\right]\right|^{2} \\
\leqslant & 2\left|\mathbb{E}_{t_{n}}^{X^{n}}\left[\Delta W_{n} e_{\nabla Y}^{n+2} \nabla_{x^{n}} X^{n+1}\right]\right|^{2} \\
& +2\left|\mathbb{E}_{t_{n}}^{X^{n}}\left[\Delta W_{n} e_{\nabla Y}^{n+2} \sum_{\alpha \in \Gamma_{2} \backslash\{v\}} \partial_{x} g_{\alpha}\left(t_{n+1}, X^{n+1}\right) I_{\alpha, n+1} \nabla_{x^{n}} X^{n+1}\right]\right|^{2} \\
\leqslant & 2 d \Delta \operatorname{Var}^{n}\left(e_{\nabla Y}^{n+2}\right)+C \Delta^{2} \mathbb{E}_{t_{n}}^{X^{n}}\left[\left|e_{\nabla Y}^{n+2}\right|^{2}\right] .
\end{aligned}
$$

Similarly we have the estimates

$$
\begin{aligned}
& \left|\mathbb{E}_{t_{n}}^{X^{n}}\left[\Delta W_{n+1} e_{\nabla Y}^{n+2} \nabla_{x^{n}} X^{n+2}\right]\right|^{2} \leqslant 2 d \Delta \mathbb{E}_{t_{n}}^{X^{n}}\left[\operatorname{Var}^{n+1}\left(e_{\nabla Y}^{n+2}\right)\right]+C \Delta^{2} \mathbb{E}_{t_{n}}^{X^{n}}\left[\left|e_{\nabla Y}^{n+2}\right|^{2}\right], \\
& \left|\mathbb{E}_{t_{n}}^{X^{n}}\left[\Delta W_{n} e_{\nabla f}^{n+1} \nabla_{x^{n}} X^{n+2}\right]\right|^{2} \leqslant C \Delta \mathbb{E}_{t_{n}}^{X^{n}}\left[\left|e_{Y}^{n+1}\right|^{2}+\left|e_{Z}^{n+1}\right|^{2}+\left|e_{\nabla Y}^{n+1}\right|^{2}+\left|e_{\nabla Z}^{n+1}\right|^{2}\right], \\
& \left|\mathbb{E}_{t_{n}}^{X^{n}}\left[\Delta W_{n} e_{\nabla f}^{n+2} \nabla_{x^{n}} X^{n+2}\right]\right|^{2} \leqslant C \Delta \mathbb{E}_{t_{n}}^{X^{n}}\left[\left|e_{Y}^{n+2}\right|^{2}+\left|e_{Z}^{n+2}\right|^{2}+\left|e_{\nabla Y}^{n+2}\right|^{2}+\left|e_{\nabla Z}^{n+2}\right|^{2}\right], \\
& \left|\mathbb{E}_{t_{n}}^{X^{n}}\left[\Delta W_{n+1} e_{\nabla f}^{n+2} \nabla_{x^{n}} X^{n+2}\right]\right|^{2} \leqslant C \Delta \mathbb{E}_{t_{n}}^{X^{n}}\left[\left|e_{Y}^{n+2}\right|^{2}+\left|e_{Z}^{n+2}\right|^{2}+\left|e_{\nabla Y}^{n+2}\right|^{2}+\left|e_{\nabla Z}^{n+2}\right|^{2}\right] .
\end{aligned}
$$

Now by (5.21), the above five estimates, and using $\left(\sum_{i=1}^{m} a_{i}\right)^{2} \leqslant m \sum_{i=1}^{m} a_{i}^{2}$, we deduce

$$
\begin{aligned}
\Delta\left|e_{\nabla Z}^{n}\right|^{2} \leqslant & C \Delta\left|\mathbb{E}_{t_{n}}^{X_{n}^{n}}\left[e_{\nabla Z}^{n+2} \nabla_{x^{n}} X^{n+2}\right]\right|^{2}+C \operatorname{Var}^{n}\left(e_{\nabla Y}^{n+2}\right)+C \mathbb{E}_{t_{n}}^{X^{n}}\left[\operatorname{Var}^{n+1}\left(e_{\nabla Y}^{n+2}\right)\right] \\
& +C \Delta \mathbb{E}_{t_{n}}^{X^{n}}\left[\left|e_{Y}^{n+2}\right|^{2}\right]+C \Delta^{2} \mathbb{E}_{t_{n}}^{X^{n}}\left[\left|e_{Y}^{n+1}\right|^{2}+\left|e_{Z}^{n+1}\right|^{2}+\left|e_{\nabla Y}^{n+1}\right|^{2}+\left|e_{\nabla Z}^{n+1}\right|^{2}\right] \\
& +C \Delta^{2} \mathbb{E}_{t_{n}}^{X^{n}}\left[\left|e_{Y}^{n+2}\right|^{2}+\left|e_{Z}^{n+2}\right|^{2}+\left|e_{\nabla Y}^{n+2}\right|^{2}+\left|e_{\nabla Z}^{n+2}\right|^{2}\right] \\
& +\frac{C}{\Delta} \sum_{j=1}^{2}\left(\left|\mathbb{E}_{t_{n}}^{X^{n}}\left[\Delta W_{n} \nabla_{x^{n}} R_{y j}^{n+1}\right]\right|^{2}+\left|\nabla_{x^{n}} R_{z j}^{n}\right|^{2}+\left|\mathbb{E}_{t_{n}}^{X^{n}}\left[\nabla_{x^{n}} R_{z j}^{n+1}\right]\right|^{2}\right) .
\end{aligned}
$$

We remain to estimate the first term on the right side of (5.22). Taking the variation $\nabla_{x^{n}}$ to $X^{n+2}$, which gives

$$
\begin{aligned}
\nabla_{x^{n}} X^{n+2} & =\nabla_{x^{n}}\left[X^{n}+\sum_{\alpha \in \Gamma_{2} \backslash\{v\}} g_{\alpha}\left(t_{n}, X^{n}\right) I_{\alpha, n}+\sum_{\alpha \in \Gamma_{2} \backslash\{v\}} g_{\alpha}\left(t_{n+1}, X^{n+1}\right) I_{\alpha, n+1}\right] \\
& =I_{d \times d}+\sum_{\alpha \in \Gamma_{2} \backslash\{v\}} \partial_{x} g_{\alpha}\left(t_{n}, X^{n}\right) I_{\alpha, n}+\sum_{\alpha \in \Gamma_{2} \backslash\{v\}} \partial_{x} g_{\alpha}\left(t_{n+1}, X^{n+1}\right) \nabla_{x^{n}} X^{n+1} I_{\alpha, n+1},
\end{aligned}
$$

and using the inequality $\left(\sum_{i=1}^{m} a_{i}\right)^{2} \leqslant m \sum_{i=1}^{m} a_{i}^{2}$, we get

$$
\left|\mathbb{E}_{t_{n}}^{X^{n}}\left[e_{\nabla Z}^{n+2} \nabla_{x^{n}} X^{n+2}\right]\right|^{2} \leqslant 3\left|\mathbb{E}_{t_{n}}^{X^{n}}\left[e_{\nabla Z}^{n+2}\right]\right|^{2}+C \Delta \mathbb{E}_{t_{n}}^{X^{n}}\left[\left|e_{\nabla Z}^{n+2}\right|^{2}\right] .
$$

Using the integration-by-parts formula of Malliavin calculus (2.9) we obtain

$$
\begin{aligned}
\mathbb{E}_{t_{n+1}}^{X^{n+1}}\left[e_{Z}^{i, n+2} \Delta W_{n+1}^{\top}\right]= & \mathbb{E}_{t_{n+1}}^{X^{n+1}}\left[e_{\nabla Z}^{i, n+2}\right] \sigma\left(t_{n+1}, X^{n+1}\right) \Delta \\
& +\sum_{\alpha \in \mathcal{A}_{2}} \mathbb{E}_{t_{n+1}}^{X^{n+1}}\left[e_{\nabla Z}^{i, n+2} g_{\alpha}\left(t_{n+1}, X^{n+1}\right) \int_{t_{n+1}}^{t_{n+2}} D_{t} I_{\alpha, n+1} d t\right] .
\end{aligned}
$$

By the definition of the norm $|\cdot|$, we have

$$
\left|e_{\nabla Z}^{n+2}\right|^{2}=\operatorname{trace}\left(\left[e_{\nabla Z}^{n+2}\right]^{\top} e_{\nabla Z}^{n+2}\right)=\sum_{i, j=1}^{d}\left|e_{\nabla Z}^{i, j, n+2}\right|^{2}=\sum_{i=1}^{d} e_{\nabla Z}^{i, n+2}\left[e_{\nabla Z}^{i, n+2}\right]^{\top},
$$


where $e_{\nabla Z}^{n+2}:=\left[e_{\nabla Z}^{i, j, n+2}\right]_{d \times d}$, and $e_{\nabla Z}^{i, n+2}:=\left(e_{\nabla Z}^{i, 1, n+2}, \ldots, e_{\nabla Z}^{i, d, n+2}\right)_{1 \times d}$ is the $i$-th row vector of $e_{\nabla Z}^{n+2}$. The uniformly elliptic condition

$$
\sigma\left(t_{n+1}, X^{n+1}\right) \sigma^{\top}\left(t_{n+1}, X^{n+1}\right) \geqslant \frac{1}{K^{\prime}} I_{d \times d}
$$

in Assumption 4.2 implies that $\sigma\left(t_{n+1}, X^{n+1}\right) \sigma^{\top}\left(t_{n+1}, X^{n+1}\right)-\frac{1}{K^{\prime}} I_{d \times d}$ is a positive semi-definite matrix, that is

$$
\mathbb{E}_{t_{n+1}}^{X^{n+1}}\left[e_{\nabla Z}^{i, n+2}\right]\left(\sigma\left(t_{n+1}, X^{n+1}\right) \sigma^{\top}\left(t_{n+1}, X^{n+1}\right)-\frac{1}{K^{\prime}} I_{d \times d}\right) \mathbb{E}_{t_{n+1}}^{X^{n+1}}\left[e_{\nabla Z}^{i, n+2}\right]^{\top} \geqslant 0,
$$

which yields

$$
\begin{aligned}
& \sum_{i=1}^{d}\left|\mathbb{E}_{t_{n+1}}^{X^{n+1}}\left[e_{\nabla Z}^{i, n+2}\right] \sigma\left(t_{n+1}, X^{n+1}\right)\right|^{2} \\
= & \sum_{i=1}^{d} \mathbb{E}_{t_{n+1}}^{X^{n+1}}\left[e_{\nabla Z}^{i, n+2}\right] \sigma\left(t_{n+1}, X^{n+1}\right) \sigma^{\top}\left(t_{n+1}, X^{n+1}\right) \mathbb{E}_{t_{n+1}}^{X^{n+1}}\left[e_{\nabla Z}^{i, n+2}\right]^{\top} \\
\geqslant & \frac{1}{K^{\prime}} \sum_{i=1}^{d} \mathbb{E}_{t_{n+1}}^{X^{n+1}}\left[e_{\nabla Z}^{i, n+2}\right] \mathbb{E}_{t_{n+1}}^{X^{n+1}}\left[e_{\nabla Z}^{i, n+2}\right]^{\top}=\frac{1}{K^{\prime}}\left|\mathbb{E}_{t_{n+1}}^{X^{n+1}}\left[e_{\nabla Z}^{n+2}\right]\right|^{2} .
\end{aligned}
$$

Thanks to the Cauchy-Schwarz inequality, it holds that

$$
\sum_{i=1}^{d}\left|\mathbb{E}_{t_{n+1}}^{X^{n+1}}\left[e_{Z}^{i, n+2} \Delta W_{n+1}^{\top}\right]\right|^{2} \leqslant d \Delta \sum_{i=1}^{d} \operatorname{Var}^{n+1}\left(e_{Z}^{i, n+2}\right)=d \Delta \operatorname{Var}^{n+1}\left(e_{Z}^{n+2}\right) .
$$

Now, using (5.24), (5.25) and (5.26) we deduce

$$
\begin{aligned}
\frac{1}{K^{\prime}} \Delta^{2}\left|\mathbb{E}_{t_{n+1}}^{X^{n+1}}\left[e_{\nabla Z}^{n+2}\right]\right|^{2} \leqslant & 2 \sum_{i=1}^{d}\left|\mathbb{E}_{t_{n+1}}^{X^{n+1}}\left[e_{Z}^{i, n+2} \Delta W_{n+1}^{\top}\right]\right|^{2} \\
& +2 \sum_{i=1}^{d} \sum_{\alpha \in \mathcal{A}_{2}}\left|\mathbb{E}_{t_{n+1}}^{X^{n+1}}\left[e_{\nabla Z}^{i, n+2} g_{\alpha}\left(t_{n+1}, X^{n+1}\right) \int_{t_{n+1}}^{t_{n+2}} D_{t} I_{\alpha, n+1} d t\right]\right|^{2} \\
\leqslant & 2 d \Delta \operatorname{Var}^{n+1}\left(e_{Z}^{n+2}\right)+C \Delta^{3} \mathbb{E}_{t_{n+1}}^{X^{n+1}}\left[\left|e_{\nabla Z}^{n+2}\right|^{2}\right],
\end{aligned}
$$

which implies

$$
\Delta\left|\mathbb{E}_{t_{n+1}}^{X^{n+1}}\left[e_{\nabla Z}^{n+2}\right]\right|^{2} \leqslant 2 K^{\prime} d \operatorname{Var}^{n+1}\left(e_{Z}^{n+2}\right)+C \Delta^{2} \mathbb{E}_{t_{n+1}}^{X^{n+1}}\left[\left|e_{\nabla Z}^{n+2}\right|^{2}\right] .
$$

By the inequality

$$
\left|\mathbb{E}_{t_{n}}^{X^{n}}\left[e_{\nabla Z}^{n+2}\right]\right|^{2}=\left|\mathbb{E}_{t_{n}}^{X^{n}}\left[\mathbb{E}_{t_{n+1}}^{X^{n+1}}\left[e_{\nabla Z}^{n+2}\right]\right]\right|^{2} \leqslant \mathbb{E}_{t_{n}}^{X^{n}}\left[\left|\mathbb{E}_{t_{n+1}}^{X^{n+1}}\left[e_{\nabla Z}^{n+2}\right]\right|^{2}\right],
$$

(5.22) and (5.27), we obtain

$$
\begin{aligned}
\Delta\left|e_{\nabla Z}^{n}\right|^{2} \leqslant & C \mathbb{E}_{t_{n}}^{X^{n}}\left[\operatorname{Var}^{n+1}\left(e_{Z}^{n+2}\right)\right]+C \operatorname{Var}^{n}\left(e_{\nabla Y}^{n+2}\right)+C \mathbb{E}_{t_{n}}^{X^{n}}\left[\operatorname{Var}^{n+1}\left(e_{\nabla Y}^{n+2}\right)\right] \\
& +C \Delta \mathbb{E}_{t_{n}}^{X^{n}}\left[\left|e_{Y}^{n+1}\right|^{2}\right]+C \Delta^{2} \mathbb{E}_{t_{n}}^{X^{n}}\left[\left|e_{Y}^{n+1}\right|^{2}+\left|e_{Z}^{n+1}\right|^{2}+\left|e_{\nabla Y}^{n+1}\right|^{2}+\left|e_{\nabla Z}^{n+1}\right|^{2}\right] \\
& +C \Delta^{2} \mathbb{E}_{t_{n}}^{X^{n}}\left[\left|e_{Y}^{n+2}\right|^{2}+\left|e_{Z}^{n+2}\right|^{2}+\left|e_{\nabla Y}^{n+2}\right|^{2}+\left|e_{\nabla Z}^{n+2}\right|^{2}\right]+\sum_{j=1}^{2} \frac{C}{\Delta}\left(\left|\mathbb{E}_{t_{n}}^{X^{n}}\left[\Delta W_{n} \nabla_{x^{n}} R_{y j}^{n+1}\right]\right|^{2}\right. \\
& \left.+\left|\nabla_{x^{n}} R_{z j}^{n}\right|^{2}+\left|\mathbb{E}_{t_{n}}^{X^{n}}\left[\nabla_{x^{n}} R_{z j}^{n+1}\right]\right|^{2}\right) .
\end{aligned}
$$

(5) The estimate (4.12) in the theorem. 
Combining the inequalities (5.4), (5.13), (5.20) and (5.28), and applying Lemma 5.0.1, we deduce

$$
\begin{aligned}
& \left|e_{Y}^{n}\right|^{2}+\left|e_{Z}^{n}\right|^{2}+\left|e_{\nabla Y}^{n}\right|^{2}+\frac{1}{4 C} \Delta\left|e_{\nabla Z}^{n}\right|^{2} \\
& \leqslant(1+C \Delta) \mathbb{E}_{t_{n}}^{X_{n}^{n}}\left[\left|\mathbb{E}_{t_{n}+1}^{X^{n+1}}\left[e_{Y}^{n+2}\right]\right|^{2}+\left|\mathbb{E}_{t_{n+1}}^{X^{n+1}}\left[e_{Z}^{n+2}\right]\right|^{2}+\left|\mathbb{E}_{t_{n}}^{X^{n}}\left[e_{\nabla Y}^{n+2}\right]\right|^{2}\right] \\
& +\left(\frac{C}{\gamma_{1}}+\frac{C}{\gamma_{2}}+\frac{C}{\gamma_{3}}\right) \mathbb{E}_{t_{n}}^{X^{n}}\left[\operatorname{Var}^{n+1}\left(e_{Y}^{n+2}\right)\right]+\frac{1}{4} \mathbb{E}_{t_{n}}^{X^{n}}\left[\operatorname{Var}^{n+1}\left(e_{Z}^{n+2}\right)\right] \\
& +\left(\frac{1}{4}+C\left(\frac{1}{\gamma_{3}}+\Delta\right)\right) \operatorname{Var}^{n}\left(e_{\nabla Y}^{n+2}\right)+\left(\frac{C}{\gamma_{2}}+\frac{C}{\gamma_{3}}+\frac{1}{4}\right) \mathbb{E}_{t_{n}}^{X^{n}}\left[\operatorname{Var}^{n+1}\left(e_{\nabla Y}^{n+2}\right)\right] \\
& +C \Delta\left(\left|e_{Y}^{n}\right|^{2}+\left|e_{Z}^{n}\right|^{2}+\left|e_{\nabla Y}^{n}\right|^{2}+\left|e_{\nabla Z}^{n}\right|^{2}\right)+C \Delta \mathbb{E}_{t_{n}}^{X^{n}}\left[\left|e_{Y}^{n+2}\right|^{2}+\left|e_{Z}^{n+2}\right|^{2}\right. \\
& \left.+\left|e_{\nabla Y}^{n+2}\right|^{2}+\left|e_{\nabla Z}^{n+2}\right|^{2}\right]+C \sum_{j=1}^{2} \frac{1}{\Delta^{3}}\left\{\left|\mathbb{E}_{t_{n}}^{X^{n}}\left[R_{y j}^{n+1} \Delta W_{n}^{\top}\right]\right|^{2}+\left|R_{z j}^{n}-\mathbb{E}_{t_{n}}^{X^{n}}\left[R_{z j}^{n+1}\right]\right|^{2}\right\} \\
& +C \sum_{j=1}^{2} \frac{1}{\Delta}\left\{\left|\mathbb{E}_{t_{n}}^{X^{n}}\left[R_{y j}^{n+1}\right]\right|^{2}+\left|\mathbb{E}_{t_{n}}^{X^{n}}\left[\nabla_{x^{n}} R_{y j}^{n+1}\right]\right|^{2}+\left|\mathbb{E}_{t_{n}}^{X^{n}}\left[\nabla_{x^{n}} R_{z j}^{n+1}\right]\right|^{2}\right. \\
& \quad+\left|\mathbb{E}_{t_{n}}^{X^{n}}\left[\Delta W_{n} \nabla_{x^{n}} R_{y j}^{n+1}\right]\right|^{2}+\left|R_{y j}^{n}\right|^{2}+\left|\nabla_{x^{n}} R_{y j}^{n}\right|^{2}+\left|\nabla_{x^{n}} R_{z j}^{n}\right|^{2} \\
& \left.\quad+\mathbb{E}_{t_{n}}^{X_{n}^{n}}\left[\left|R_{z j}^{n+1}\right|^{2}\right]+\mathbb{E}_{t_{n}}^{X^{n}}\left[\left|\nabla_{x^{n+1}} R_{z j}^{n+1}\right|^{2}\right]\right\} .
\end{aligned}
$$

Notice that

$$
\begin{aligned}
& \left|\mathbb{E}_{t_{n+1}}^{X^{n+1}}\left[e_{Y}^{n+2}\right]\right|^{2}+\frac{3}{8} \operatorname{Var}^{n+1}\left(e_{Y}^{n+2}\right)=\frac{5}{8}\left|\mathbb{E}_{t_{n+1}}^{X^{n+1}}\left[e_{Y}^{n+2}\right]\right|^{2}+\frac{3}{8} \mathbb{E}_{t_{n+1}}^{X^{n+1}}\left[\left|e_{Y}^{n+2}\right|^{2}\right] \leqslant \mathbb{E}_{t_{n+1}}^{X^{n+1}}\left[\left|e_{Y}^{n+2}\right|^{2}\right], \\
& \left|\mathbb{E}_{t_{n+1}}^{X^{n+1}}\left[e_{Z}^{n+2}\right]\right|^{2}+\frac{1}{4} \operatorname{Var}^{n+1}\left(e_{Z}^{n+2}\right)=\frac{3}{4}\left|\mathbb{E}_{t_{n+1}}^{X^{n+1}}\left[e_{Z}^{n+2}\right]\right|^{2}+\frac{1}{4} \mathbb{E}_{t_{n+1}}^{X^{n+1}}\left[\left|e_{Z}^{n+2}\right|^{2}\right] \leqslant \mathbb{E}_{t_{n+1}}^{X^{n+1}}\left[\left|e_{Z}^{n+2}\right|^{2}\right], \\
& \left|\mathbb{E}_{t_{n}}^{X^{n}}\left[e_{\nabla Y}^{n+2}\right]\right|^{2}+\left(\frac{1}{4}+\varsigma\right) \operatorname{Var}^{n}\left(e_{\nabla Y}^{n+2}\right)+\frac{1}{2} \mathbb{E}_{t_{n}}^{X^{n}}\left[\operatorname{Var}^{n+1}\left(e_{\nabla Y}^{n+2}\right)\right] \\
& \leqslant\left(\frac{1}{4}+\varsigma\right)\left|\mathbb{E}_{t_{n}}^{X^{n}}\left[e_{\nabla Y}^{n+2}\right]\right|^{2}+\left(\frac{3}{4}-\varsigma\right) \mathbb{E}_{t_{n}}^{X^{n}}\left[\left|\mathbb{E}_{t_{n+1}}^{X^{n+1}}\left[e_{\nabla Y}^{n+2}\right]\right|^{2}\right] \\
& \quad+\left(\frac{1}{4}+\varsigma\right) \operatorname{Var}^{n}\left(e_{\nabla Y}^{n+2}\right)+\frac{1}{2} \mathbb{E}_{t_{n}}^{X^{n}}\left[\operatorname{Var}^{n+1}\left(e_{\nabla Y}^{n+2}\right)\right] \\
& \leqslant\left(\left(\frac{1}{4}+\varsigma\right)+\frac{1}{2}\right) \mathbb{E}_{t_{n}}^{X^{n}}\left[\left|e_{\nabla Y}^{n+2}\right|^{2}\right]+\left(\frac{1}{4}-\varsigma\right) \mathbb{E}_{t_{n}}^{X^{n}}\left[\left|\mathbb{E}_{t_{n+1}}^{X^{n+1}}\left[e_{\nabla Y}^{n+2}\right]\right|^{2}\right] \leqslant \mathbb{E}_{t_{n}}^{X^{n}}\left[\left|e_{\nabla Y}^{n+2}\right|^{2}\right], \quad 0<\varsigma<\frac{1}{4} .
\end{aligned}
$$

Now let $\gamma_{1}=\gamma_{2}=\gamma_{3}=8 C$ and $\Delta_{0}$ be sufficient small such that $0<C\left(\frac{1}{\gamma_{3}}+\Delta\right)<\frac{1}{4}$ for $\Delta<\Delta_{0}$. Then, by inequality (5.29), we deduce

$$
\begin{gathered}
\left|e_{Y}^{n}\right|^{2}+\left|e_{Z}^{n}\right|^{2}+\left|e_{\nabla Y}^{n}\right|^{2}+\frac{1}{4 C} \Delta\left|e_{\nabla Z}^{n}\right|^{2} \\
\leqslant \frac{1+C \Delta}{1-C \Delta} \mathbb{E}_{t_{n}}^{X_{n}}\left[\left|e_{Y}^{n+2}\right|^{2}+\left|e_{Z}^{n+2}\right|^{2}+\left|e_{\nabla Y}^{n+2}\right|^{2}+\frac{1}{4 C} \Delta\left|e_{\nabla Z}^{n+2}\right|^{2}\right] \\
+C \sum_{j=1}^{2} \frac{1}{\Delta^{3}}\left\{\left|\mathbb{E}_{t_{n}}^{X^{n}}\left[R_{y j}^{n+1} \Delta W_{n}^{\top}\right]\right|^{2}+\left|R_{z j}^{n}-\mathbb{E}_{t_{n}}^{X^{n}}\left[R_{z j}^{n+1}\right]\right|^{2}\right\} \\
+C \sum_{j=1}^{2} \frac{1}{\Delta}\left\{\left|\mathbb{E}_{t_{n}}^{X^{n}}\left[R_{y j}^{n+1}\right]\right|^{2}+\left|\mathbb{E}_{t_{n}}^{X^{n}}\left[\nabla_{x^{n}} R_{y j}^{n+1}\right]\right|^{2}+\left|\mathbb{E}_{t_{n}}^{X^{n}}\left[\nabla_{x^{n}} R_{z j}^{n+1}\right]\right|^{2}\right. \\
+\left|\mathbb{E}_{t_{n}}^{X_{n}^{n}}\left[\Delta W_{n} \nabla_{x^{n}} R_{y j}^{n+1}\right]\right|^{2}+\left|R_{y j}^{n}\right|^{2}+\left|\nabla_{x^{n}} R_{y j}^{n}\right|^{2}+\left|\nabla_{x^{n}} R_{z j}^{n}\right|^{2} \\
\left.+\mathbb{E}_{t_{n}}^{X^{n}}\left[\left|R_{z j}^{n+1}\right|^{2}\right]+\mathbb{E}_{t_{n}}^{X_{n}^{n}}\left[\left|\nabla_{x^{n+1}} R_{z j}^{n+1}\right|^{2}\right]\right\} \\
\leqslant e^{C T} \mathbb{E}_{t_{n}}^{X^{n}}\left[\left|e_{Y}^{N-1}\right|^{2}+\left|e_{Z}^{N-1}\right|^{2}+\left|e_{\nabla Y}^{N-1}\right|^{2}+\frac{1}{4 C} \Delta\left|e_{\nabla Z}^{N-1}\right|^{2}\right] \\
+C \sum_{i=n}^{N-2} \sum_{j=1}^{2}\left\{\frac{1}{\Delta^{3}}\left(\left|\mathbb{E}_{t_{i}}^{X^{i}}\left[R_{y j}^{i+1} \Delta W_{i}^{\top}\right]\right|^{2}+\left|R_{z j}^{i}-\mathbb{E}_{t_{i}}^{X^{i}}\left[R_{z j}^{i+1}\right]\right|^{2}\right)\right. \\
+\frac{1}{\Delta}\left(\left|\mathbb{E}_{t_{i}}^{X^{i}}\left[R_{y j}^{i+1}\right]\right|^{2}+\left|\mathbb{E}_{t_{i}}^{X^{i}}\left[\nabla_{x^{i}} R_{y j}^{i+1}\right]\right|^{2}+\left|\mathbb{E}_{t_{i}}^{X^{i}}\left[\nabla_{x^{i}} R_{z j}^{i+1}\right]\right|^{2}\right. \\
+\left|\mathbb{E}_{t_{i}}^{X^{i}}\left[\Delta W_{i} \nabla_{x^{i}} R_{y j}^{i+1}\right]\right|^{2}+\left|R_{y j}^{i}\right|^{2}+\left|\nabla_{x^{i}} R_{y j}^{i}\right|^{2}+\left|\nabla_{x^{i}} R_{z j}^{i}\right|^{2} \\
\left.\left.+\mathbb{E}_{t_{i}}^{X^{i}}\left[\left|R_{z j}^{i+1}\right|^{2}\right]+\mathbb{E}_{t_{i}}^{X^{i}}\left[\left|\nabla_{x^{i+1}} R_{z j}^{i+1}\right|^{2}\right]\right)\right\}
\end{gathered}
$$

And then by taking the mathematical expectation $\mathbb{E}[\cdot]$ on both sides of (5.30), we complete the proof.

Remark 5.1. The remainder terms include three types: (1) the truncation error terms, e.g., $R_{y 1}^{i}$ and $\nabla_{x^{i}} R_{y 1}^{i}$; (2) the discretization errors caused by the discretization of SDE, e.g., $R_{y 2}^{i}$ and $\nabla_{x^{i}} R_{y 2}^{i}$; (3) the error terms in (2) multiplied by $\Delta W_{i}^{\top}$ (e.g., $\mathbb{E}_{t_{i}}^{X^{i}}\left[R_{y j}^{i+1} \Delta W_{i}^{\top}\right]$ ). Under certain regularity conditions on the data $b, \sigma, f$ and $\varphi$, by the Itô-Taylor and Taylor expansion, and the Malliavin calculus, we can obtain the estimates of these remainder terms (which are proved in detail in Section 5.2). Subsequently, it is easy to get error estimates for Scheme 3.1 by Theorem 4.1 . 


\subsection{Proof of Theorem 4.2}

We consider the case that the generator $f$ of FBSDEs (1.1) is a deterministic function.

\subsubsection{Useful lemmas}

In this subsection, we introduce some lemmas which will be used in the proof of Theorem 4.2 They may also be very useful in error analysis for other numerical methods for solving FBSDEs.

Lemma 5.2.1. For $X^{n+1}=\sum_{\alpha \in \Gamma_{2}} g_{\alpha}\left(t_{n}, X^{n}\right) I_{\alpha, n}$, if $b, \sigma \in C_{b}^{2,5}$ and $H \in C_{b}^{5}$, then under Hypothesis 4.1 , for $1 \leqslant n \leqslant N-2$, there exists a positive integer $q$ such that

$$
\left|\nabla_{x^{n}} \mathbb{E}_{t_{n}}^{X^{n}}\left[H\left(X_{t_{n+1}}^{t_{n}, X^{n}}\right)-H\left(X^{n+1}\right)\right]\right| \leqslant C\left(1+\left|X^{n}\right|^{q}\right) \Delta^{3},
$$

where $C$ is a positive constant depending on $K$, and upper bounds of the derivatives of $b, \sigma$ and $H$.

Proof. For $0 \leqslant n \leqslant N-2$, using the multiple Taylor expansion, we obtain

$$
\mathbb{E}_{t_{n}}^{X^{n}}\left[H\left(X_{t_{n+1}}^{t_{n}, X^{n}}\right)-H\left(X^{n+1}\right)\right]=\sum_{i=1}^{d} \mathbb{E}_{t_{n}}^{X^{n}}\left[h_{n}^{i} F_{x_{i}}^{X^{n}}\right],
$$

where $h_{n}^{i}=\sum_{\alpha \in \mathcal{A}_{3}} I_{\alpha}\left[g_{\alpha}^{i}\left(\cdot, X^{t_{n}, X^{n}}\right)\right]_{t_{n}, t_{n+1}}$ and $F_{x_{i}}^{X^{n}}=\int_{0}^{1} H_{x_{i}}^{\prime}\left(X^{n+1}+\lambda\left(X_{t_{n+1}}^{t_{n}, X^{n}}-X^{n+1}\right)\right) d \lambda$. Then under the assumption $b, \sigma \in C_{b}^{2,5}$ and $H \in C_{b}^{5}$, by the integration-by-parts formula (2.9) of Malliavin calculus and inequality (4.3), we deduce

$$
\begin{aligned}
& \left|\nabla_{x^{n}} \mathbb{E}_{t_{n}}^{X^{n}}\left[H\left(X_{t_{n+1}}^{t_{n}, X^{n}}\right)-H\left(X^{n+1}\right)\right]\right|=\left|\sum_{\alpha \in \mathcal{A}_{3}} \sum_{i=1}^{d} \nabla_{x^{n}} \mathbb{E}_{t_{n}}^{X^{n}}\left[h_{n}^{i} F_{x_{i}}^{X^{n}}\right]\right| \\
= & \left|\sum_{\alpha \in \mathcal{A}_{3}} \sum_{i=1}^{d} \int_{t_{n}}^{t_{n+1}} \int_{t_{n}}^{s_{3}} \int_{t_{n}}^{s_{2}} \mathbb{E}_{t_{n}}^{X^{n}}\left[\nabla_{x^{n}}\left\{D_{s_{1} s_{2} s_{3}}^{\alpha}\left(F_{x_{i}}^{X^{n}}\right) g_{\alpha}^{i}\left(s_{1}, X_{s_{1}}^{t_{n}, X^{n}}\right)\right\}\right] d s_{1} d s_{2} d s_{3}\right| \\
\leqslant & C\left(1+\left|X^{n}\right|^{q}\right) \Delta^{3} .
\end{aligned}
$$

The proof is competed.

Lemma 5.2.2. If $b, \sigma \in C_{b}^{2,4}$ and $H \in C_{b}^{3,6}$, then under Hypothesis 4.1 for $1 \leqslant n \leqslant N-2$, there exists a generic positive integer $q$ such that

$$
\left|\mathbb{E}_{t_{n}}^{X^{n}}\left[\Delta W_{n} R_{n+1}^{n+2}\right]\right| \leqslant C\left(1+\left|X^{n}\right|^{q}\right) \Delta^{4},
$$

moreover, if $b, \sigma \in C_{b}^{2,5}$ and $H \in C_{b}^{3,7}$, then

$$
\left|\mathbb{E}_{t_{n}}^{X^{n}}\left[\Delta W_{n} \nabla_{x^{n}} R_{n+1}^{n+2}\right]\right| \leqslant C\left(1+\left|X^{n}\right|^{q}\right) \Delta^{4}
$$

where $R_{n+1}^{n+2}=\mathbb{E}_{t_{n+1}}^{X^{n+1}}\left[\int_{t_{n+1}}^{t_{n+1}}\left\{H\left(t, X_{t}^{t_{n+1}, X^{n+1}}\right)-\frac{H\left(t_{n+1}, X^{n+1}\right)+H\left(t_{n+2}, X_{t_{n+2}}^{t_{n+1}, X^{n+1}}\right)}{2}\right\} d t\right]$, and $C$ is a positive constant depending on $K$, and upper bounds of the derivatives of $b, \sigma$ and $H$.

Proof. Since $\Delta W_{n}$ is $\mathcal{F}_{t_{n+1}}$-measurable increment, we have the identity

$$
\begin{aligned}
& \mathbb{E}_{t_{n}}^{X^{n}}\left[\Delta W_{n} \int_{t_{n+1}}^{t_{n+2}}\left\{H\left(t, X_{t}^{t_{n+1}, X^{n+1}}\right)-\frac{H\left(t_{n+1}, X^{n+1}\right)+H\left(t_{n+2}, X_{t_{n+2}}^{t_{n+1}, X^{n+1}}\right)}{2}\right\} d t\right] \\
= & \mathbb{E}_{t_{n}}^{X^{n}}\left[\Delta W_{n} \mathbb{E}_{t_{n+1}}^{X^{n+1}}\left[\int_{t_{n+1}}^{t_{n+2}}\left\{H\left(t, X_{t}^{t_{n+1}, X^{n+1}}\right)-\frac{H\left(t_{n+1}, X^{n+1}\right)+H\left(t_{n+2}, X_{t_{n+2}}^{t_{n+1}, X^{n+1}}\right)}{2}\right\} d t\right] .\right.
\end{aligned}
$$


The Itô formula then shows that

$$
\begin{aligned}
H\left(t, X_{t}^{t_{n+1}, X^{n+1}}\right)= & H\left(t_{n+1}, X^{n+1}\right)+\int_{t_{n+1}}^{t} L^{0} H\left(s, X_{s}^{t_{n+1}, X^{n+1}}\right) d s \\
& +\sum_{j=1}^{d} \int_{t_{n+1}}^{t} L^{j} H\left(s, X_{s}^{t_{n+1}, X^{n+1}}\right) d W_{s}^{j}, \\
L^{0} H\left(s, X_{s}^{\left.t_{n+1}, X^{n+1}\right)=}\right. & L^{0} H\left(t_{n+1}, X^{n+1}\right)+\int_{t_{n+1}}^{s} L^{0} L^{0} H\left(\tau, X_{\tau}^{t_{n+1}, X^{n+1}}\right) d \tau \\
& +\sum_{j=1}^{d} \int_{t_{n+1}}^{s} L^{j} L^{0} H\left(\tau, X_{\tau}^{t_{n+1}, X^{n+1}}\right) d W_{\tau}^{j}, \\
L^{0} L^{0} H\left(\tau, X_{\tau}^{\left.t_{n+1}, X^{n+1}\right)=}\right. & L^{0} L^{0} H\left(t_{n+1}, X^{n+1}\right)+\int_{t_{n+1}}^{\tau} L^{0} L^{0} L^{0} H\left(\nu, X_{\nu}^{t_{n+1}, X^{n+1}}\right) d \nu \\
& +\sum_{j=1}^{d} \int_{t_{n+1}}^{\tau} L^{j} L^{0} L^{0} H\left(\nu, X_{\nu}^{t_{n+1}, X^{n+1}}\right) d W_{\nu}^{j} .
\end{aligned}
$$

By the equalities in (5.36), we have

$$
\begin{aligned}
& \mathbb{E}_{t_{n+1}}^{X^{n+1}}\left[\int_{t_{n+1}}^{t_{n+2}} H\left(t, X_{t}^{t_{n+1}, X^{n+1}}\right) d t\right] \\
= & H\left(t_{n+1}, X^{n+1}\right) \Delta+\frac{1}{2} L^{0} H\left(t_{n+1}, X^{n+1}\right) \Delta^{2}+\frac{1}{6} L^{0} L^{0} H\left(t_{n+1}, X^{n+1}\right) \Delta^{3} \\
& +\int_{t_{n+1}}^{t_{n+2}} \int_{t_{n+1}}^{t} \int_{t_{n+1}}^{s} \int_{t_{n+1}}^{\tau} \mathbb{E}_{t_{n+1}}^{X^{n+1}}\left[L^{0} L^{0} L^{0} H\left(\nu, X_{\nu}^{t_{n+1}, X^{n+1}}\right)\right] d \nu d \tau d s d t,
\end{aligned}
$$

and

$$
\begin{aligned}
& -\frac{1}{2} \int_{t_{n+1}}^{t_{n+2}} \mathbb{E}_{t_{n+1}}^{X^{n+1}}\left[H\left(t_{n+2}, X_{t_{n+2}}^{t_{n+1}, X^{n+1}}\right)\right] d t \\
= & -\frac{1}{2} H\left(t_{n+1}, X^{n+1}\right) \Delta-\frac{1}{2} L^{0} H\left(t_{n+1}, X^{n+1}\right) \Delta^{2}-\frac{1}{4} L^{0} L^{0} H\left(t_{n+1}, X^{n+1}\right) \Delta^{3} \\
& -\frac{1}{2} \int_{t_{n+1}}^{t_{n+2}} \int_{t_{n+1}}^{t_{n+2}} \int_{t_{n+1}}^{s} \int_{t_{n+1}}^{\tau} \mathbb{E}_{t_{n+1}}^{X^{n+1}}\left[L^{0} L^{0} L^{0} H\left(\nu, X_{\nu}^{t_{n+1}, X^{n+1}}\right)\right] d \nu d \tau d s d t .
\end{aligned}
$$

Then by the above two identities, we deduce

$$
\begin{aligned}
& \mathbb{E}_{t_{n}}^{X^{n}}\left[\Delta W_{n} R_{n+1}^{n+2}\right] \\
= & -\frac{1}{12} \mathbb{E}_{t_{n}}^{X^{n}}\left[\Delta W_{n} L^{0} L^{0} H\left(t_{n+1}, X^{n+1}\right)\right] \Delta^{3} \\
& +\int_{t_{n+1}}^{t_{n+1}} \int_{t_{n+1}}^{t} \int_{t_{n+1}}^{s} \int_{t_{n+1}}^{\tau} \mathbb{E}_{t_{n}}^{X^{n}}\left[\Delta W_{n} L^{0} L^{0} L^{0} H\left(\nu, X_{\nu}^{t_{n+1}, X^{n+1}}\right)\right] d \nu d \tau d s d t \\
& -\frac{1}{2} \int_{t_{n+1}}^{t_{n+2}} \int_{t_{n+1}}^{t_{n+2}} \int_{t_{n+1}}^{s} \int_{t_{n+1}}^{\tau} \mathbb{E}_{t_{n}}^{X^{n}}\left[\Delta W_{n} L^{0} L^{0} L^{0} H\left(\nu, X_{\nu}^{t_{n+1}, X^{n+1}}\right)\right] d \nu d \tau d s d t .
\end{aligned}
$$

From the Malliavin integration-by-parts formula (2.9) we deduce

$$
\mathbb{E}_{t_{n}}^{X^{n}}\left[\Delta W_{n} L^{0} L^{0} H\left(t_{n+1}, X^{n+1}\right)\right]=\int_{t_{n}}^{t_{n+1}} \mathbb{E}_{t_{n}}^{X^{n}}\left[D_{s} L^{0} L^{0} H\left(t_{n+1}, X^{n+1}\right)\right] d s .
$$

Now, under the assumptions of the lemma, combining (5.35), (5.37) and (5.38) we easily obtain the inequality (5.33). Similarly we can prove the inequality (5.34). The proof is completed.

Lemma 5.2.3. Suppose $b, \sigma \in C_{b}^{2,4}$ and $H \in C_{b}^{3,6}$, then under Hypotheses 4.1 , for $0 \leqslant n \leqslant N-2$, there exists a positive integer $q$ such that

$$
\left|\mathbb{E}_{t_{n}}^{X^{n}}\left[R_{n}^{n+1}-R_{n+1}^{n+2}\right]\right| \leqslant C\left(1+\left|X^{n}\right|^{q}\right) \Delta^{4},
$$

where $R_{n}^{n+1}=\mathbb{E}_{t_{n}}^{X^{n}}\left[\int_{t_{n}}^{t_{n+1}}\left\{H\left(t, X_{t}^{t_{n}, X^{n}}\right)-\frac{H\left(t_{n}, X^{n}\right)+H\left(t_{n+1}, X_{t_{n+1}}^{t_{n}, X^{n}}\right)}{2}\right\} d t\right]$, and $C$ is a positive constant depending on $K$, and upper bounds of the derivatives of $b, \sigma$ and $H$.

Proof. Similar to get (5.37), we have the following two equalities:

$$
\begin{aligned}
& \mathbb{E}_{t_{n}}^{X_{n}^{n}}\left[R_{n}^{n+1}\right] \\
= & -\frac{1}{12} L^{0} L^{0} H\left(t_{n}, X^{n}\right) \Delta^{3}+\int_{t_{n}}^{t_{n+1}} \int_{t_{n}}^{t} \int_{t_{n}}^{s} \int_{t_{n}}^{r} \mathbb{E}_{t_{n}}^{X^{n}}\left[L^{0} L^{0} L^{0} H\left(\tau, X_{\tau}^{t_{n}, X^{n}}\right)\right] d \tau d r d s d t \\
& -\frac{1}{2} \int_{t_{n}}^{t_{n+1}} \int_{t_{n}}^{t_{n+1}} \int_{t_{n}}^{s} \int_{t_{n}}^{r} \mathbb{E}_{t_{n}}^{X_{n}^{n}}\left[L^{0} L^{0} L^{0} H\left(\tau, X_{\tau}^{t_{n}, X^{n}}\right)\right] d \tau d r d s d t,
\end{aligned}
$$


and

$$
\begin{aligned}
& \mathbb{E}_{t_{n+1}}^{X^{n+1}}\left[R_{n+1}^{n+2}\right] \\
= & -\frac{1}{12} L^{0} L^{0} H\left(t_{n+1}, X^{n+1}\right) \Delta^{3}+\int_{t_{n+1}}^{t_{n+2}} \int_{t_{n+1}}^{t} \int_{t_{n+1}}^{s} \int_{t_{n+1}}^{r} \mathbb{E}_{t_{n+1}}^{X^{n+1}}\left[L^{0} L^{0} L^{0} H\left(\tau, X_{\tau}^{t_{n+1}, X^{n+1}}\right)\right] d \tau d r d s d t \\
& -\frac{1}{2} \int_{t_{n+1}}^{t_{n+2}} \int_{t_{n+1}}^{t_{n+2}} \int_{t_{n+1}}^{s} \int_{t_{n+1}}^{r} \mathbb{E}_{t_{n+1}}^{X^{n+1}}\left[L^{0} L^{0} L^{0} H\left(\tau, X_{\tau}^{t_{n+1}, X^{n+1}}\right)\right] d \tau d r d s d t .
\end{aligned}
$$

Now, under the conditions of the lemma and from the above two equations, we deduce

$$
\begin{aligned}
\left|\mathbb{E}_{t_{n}}^{X^{n}}\left[R_{n}^{n+1}-R_{n+1}^{n+2}\right]\right| & \leqslant \frac{1}{12} \Delta^{3}\left|\mathbb{E}_{t_{n}}^{X^{n}}\left[L^{0} L^{0} H\left(t_{n+1}, X^{n+1}\right)-L^{0} L^{0} H\left(t_{n}, X^{n}\right)\right]\right|+C\left(1+\left|X^{n}\right|^{q}\right) \Delta^{4} \\
& \leqslant C\left(1+\left|X^{n}\right|^{q}\right) \Delta^{4} .
\end{aligned}
$$

We complete the proof.

Lemma 5.2.4. For $X^{n+1}=\sum_{\alpha \in \Gamma_{2}} g_{\alpha}\left(t_{n}, X^{n}\right) I_{\alpha, n}$, if $b, \sigma \in C_{b}^{2,4}$ and $H \in C_{b}^{5}$. then under Hypothesis 4.1 , for $1 \leqslant n \leqslant N-2$, there exists a positive generic integer $q$ such that

$$
\left|\mathbb{E}_{t_{n}}^{X^{n}}\left[\Delta W_{n} U_{n+1}^{n+2}\right]\right| \leqslant C\left(1+\left|X^{n}\right|^{q}\right) \Delta^{4},
$$

moreover, if $b, \sigma \in C_{b}^{2,5}$ and $H \in C_{b}^{6}$, then

$$
\left|\mathbb{E}_{t_{n}}^{X^{n}}\left[\Delta W_{n} \nabla_{x^{n}} U_{n+1}^{n+2}\right]\right| \leqslant C\left(1+\left|X^{n}\right|^{q}\right) \Delta^{4},
$$

where $U_{n+1}^{n+2}=\mathbb{E}_{t_{n+1}}^{X_{n+1}}\left[H\left(X_{t_{n+2}}^{t_{n+1}, X^{n+1}}\right)-H\left(X^{n+2}\right)\right]$, and $C$ is a positive constant depending on $K$, and upper bounds of the derivatives of $b, \sigma$ and $H$.

Proof. The Taylor expansion shows that

$$
H\left(X_{t_{n+2}}^{t_{n+1}, X^{n+1}}\right)-H\left(X^{n+2}\right)=\sum_{i=1}^{d} h_{n+1}^{i} F_{x_{i}}^{X^{n+1}},
$$

where $h_{n+1}^{i}=\sum_{\alpha \in \mathcal{A}_{3}} I_{\alpha}\left[g_{\alpha}^{i}\left(\cdot, X^{t_{n+1}, X^{n+1}}\right)\right]_{t_{n+1}, t_{n+2}}$ and $F_{x_{i}}^{X^{n+1}}=\int_{0}^{1} H_{x_{i}}^{\prime}\left(X^{n+2}+\lambda\left(X_{t_{n+2}}^{t_{n+1}, X^{n+1}}-X^{n+2}\right)\right) d \lambda$. Now, under the conditions of the lemma, using the integration-by-parts formula of Malliavin calculus (2.9), we have

$$
\begin{aligned}
U_{n+1}^{n+2} & =\mathbb{E}_{t_{n+1}}^{X^{n+1}}\left[H\left(X_{t_{n+2}}^{t_{n+1}, X^{n+1}}\right)-H\left(X^{n+2}\right)\right] \\
& =\sum_{\alpha \in \mathcal{A}_{3}} \sum_{i=1}^{d} \mathbb{E}_{t_{n+1}}^{X^{n+1}}\left[F_{x_{i}}^{X^{n+1}} I_{\alpha}\left[g_{\alpha}^{i}\left(\cdot, X^{t_{n+1}, X^{n+1}}\right)\right]_{t_{n+1}, t_{n+2}}\right] \\
& =\sum_{\alpha \in \mathcal{A}_{3}} \sum_{i=1}^{d} \mathbb{E}_{t_{n+1}}^{X^{n+1}}\left[I_{(0,0,0)}\left[D_{s_{1} s_{2} s_{3}}^{\alpha}\left\{F_{x_{i}}^{X^{n+1}}\right\} g_{\alpha}^{i}\left(s_{1}, X_{s_{1}}^{t_{n+1}, X^{n+1}}\right)\right]_{t_{n+1}, t_{n+2}}\right] .
\end{aligned}
$$

By the integration-by-parts of Malliavin calculus (2.9) again we deduce

$$
\begin{aligned}
& \left|\mathbb{E}_{t_{n}}^{X^{n}}\left[\Delta W_{n} U_{n+1}^{n+2}\right]\right| \\
= & \left|\sum_{\alpha \in \mathcal{A}_{3}} \sum_{i=1}^{d} \mathbb{E}_{t_{n}}^{X^{n}}\left[\Delta W_{n} \mathbb{E}_{t_{n+1}}^{X^{n+1}}\left[I_{(0,0,0)}\left[D_{s_{1} s_{2} s_{3}}^{\alpha}\left\{F_{x_{i}}^{X^{n+1}}\right\} g_{\alpha}^{i}\left(s_{1}, X_{s_{1}}^{t_{n+1}, X^{n+1}}\right)\right]_{t_{n+1}, t_{n+2}}\right]\right]\right| \\
= & \left|\sum_{\alpha \in \mathcal{A}_{3}} \sum_{i=1}^{d} \mathbb{E}_{t_{n}}^{X^{n}}\left[I_{(0,0,0)}\left[\left(W_{s_{1}}-W_{t_{n}}\right) D_{s_{1} s_{2} s_{3}}^{\alpha}\left\{F_{x_{i}}^{X^{n+1}}\right\} g_{\alpha}^{i}\left(s_{1}, X_{s_{1}}^{t_{n+1}, X^{n+1}}\right)\right]_{t_{n+1}, t_{n+2}}\right]\right| \\
= & \left|\sum_{\alpha \in \mathcal{A}_{3}} \sum_{i=1}^{d} \int_{t_{n+1}}^{t_{n+2}} \int_{t_{n+1}}^{s_{3}} \int_{t_{n+1}}^{s_{2}} \int_{t_{n}}^{s_{1}} \mathbb{E}_{t_{n}}^{X^{n}}\left[D_{s}\left\{D_{s_{1} s_{2} s_{3}}^{\alpha}\left\{F_{x_{i}}^{X^{n+1}}\right\} g_{\alpha}^{i}\left(s_{1}, X_{s_{1}}^{t_{n+1}, X^{n+1}}\right)\right\}\right] d s d s_{1} d s_{2} d s_{3}\right| \\
\leqslant & C\left(1+\left|X^{n}\right|^{q}\right) \Delta^{4},
\end{aligned}
$$

which proves (5.39). The estimate (5.40) can be similarly proved. The proof is complete. 
Lemma 5.2.5. For $X^{n+1}=\sum_{\alpha \in \Gamma_{2}} g_{\alpha}\left(t_{n}, X^{n}\right) I_{\alpha, n}$, if $b, \sigma \in C_{b}^{3,6}, H \in C_{b}^{5}$, then under Hypotheses 4.1, for $1 \leqslant n \leqslant N-2$, there exists a generic positive integer $q$ such that

$$
\left|U_{n}^{n+1}-\mathbb{E}_{t_{n}}^{X^{n}}\left[U_{n+1}^{n+2}\right]\right| \leqslant C\left(1+\left|X^{n}\right|^{q}\right) \Delta^{4}
$$

for $1 \leqslant n \leqslant N-2$, where $U_{n}^{n+1}=\mathbb{E}_{t_{n}}^{X^{n}}\left[H\left(X_{t_{n+1}}^{t_{n}, X^{n}}\right)-H\left(X^{n+1}\right)\right]$, and $C$ is a positive constant depending on $K$, and upper bounds of the derivatives of $b, \sigma$ and $H$.

Proof. By the multiple Taylor expansion and the definition of $U_{n}^{n+1}$, we know

$$
\begin{aligned}
\left|U_{n}^{n+1}-\mathbb{E}_{t_{n}}^{X^{n}}\left[U_{n+1}^{n+2}\right]\right| & =\left|\mathbb{E}_{t_{n}}^{X^{n}}\left[H\left(X_{t_{n+1}}^{t_{n}, X^{n}}\right)-H\left(X^{n+1}\right)-\mathbb{E}_{t_{n+1}}^{X^{n+1}}\left[H\left(X_{t_{n+2}}^{t_{n+1}, X^{n+1}}\right)-H\left(X^{n+2}\right)\right]\right]\right| \\
& =\sum_{i=1}^{d} \mathbb{E}_{t_{n}}^{X^{n}}\left[h_{n}^{i} F_{x_{i}}^{X^{n}}\right]-\sum_{i=1}^{d} \mathbb{E}_{t_{n+1}}^{X^{n+1}}\left[h_{n+1}^{i} F_{x_{i}}^{X^{n}+1}\right],
\end{aligned}
$$

where $h_{n}^{i}=\sum_{\alpha \in \mathcal{A}_{3}} I_{\alpha}\left[g_{\alpha}^{i}\left(\cdot, X^{t_{n}, X^{n}}\right)\right]_{t_{n}, t_{n+1}}$ and $F_{x_{i}}^{X^{n}}=\int_{0}^{1} H_{x_{i}}^{\prime}\left(X^{n+1}+\lambda\left(X_{t_{n+1}}^{t_{n}, X^{n}}-X^{n+1}\right)\right) d \lambda$. Thus, by the integration-by-parts formula (2.9) of Malliavin calculus, we have

$$
\begin{aligned}
\sum_{i=1}^{d} \mathbb{E}_{t_{n}}^{X^{n}}\left[F_{x_{i}}^{X^{n}} h_{i}^{n}\right]= & \sum_{i=1}^{d} \mathbb{E}_{t_{n}}^{X^{n}}\left[\sum_{\alpha \in \mathcal{A}_{3}} F_{x_{i}}^{X^{n}} I_{\alpha, n} g_{\alpha}^{i}\left(t_{n}, X^{n}\right)+\sum_{\alpha \in \mathcal{A}_{4}} F_{x_{i}}^{X^{n}} I_{\alpha}\left[g_{\alpha}^{i}\left(\cdot, X^{t_{n}, X^{n}}\right)\right]_{t_{n}, t_{n+1}}\right] \\
= & \sum_{i=1}^{d} \sum_{\alpha \in \mathcal{A}_{3}} I_{(0,0,0)}\left[\mathbb{E}_{t_{n}}^{X^{n}}\left[D_{s_{1} s_{2} s_{3}}^{\alpha}\left\{F_{x_{i}}^{X^{n}}\right\}\right]\right]_{t_{n}, t_{n+1}} g_{\alpha}^{i}\left(t_{n}, X^{n}\right) \\
& +\sum_{i=1}^{d} \sum_{\alpha \in \mathcal{A}_{4}} I_{(0,0,0,0)}\left[\mathbb{E}_{t_{n}}^{X^{n}}\left[D_{s_{1} s_{2} s_{3} s_{4}}^{\alpha}\left\{F_{x_{i}}^{X^{n}}\right\} g_{\alpha}^{i}\left(s_{1}, X_{s_{1}}^{\left.t_{n}, X^{n}\right)}\right)\right]\right]_{t_{n}, t_{n+1}} .
\end{aligned}
$$

For $\lambda \in(0,1]$, we assume $\psi_{n}=\left(\psi_{n, 1}, \psi_{n, 2}, \ldots, \psi_{n, d}\right):=X^{n+1}-X^{n}+\lambda\left(X_{t_{n+1}}^{t_{n}, X^{n}}-X^{n+1}\right)$ with its $i$-th component $\psi_{n, i}=\phi_{n}^{i}+\lambda h_{n}^{i}(1 \leqslant i \leqslant d)$, then by the Taylor expansion, we deduce

$$
\begin{aligned}
F_{x_{i}}^{X^{n}} & =\int_{0}^{1} H_{x_{i}}^{\prime}\left(X^{n+1}+\lambda\left(X_{t_{n+1}}^{t_{n}, X^{n}}-X^{n+1}\right)\right) d \lambda=\int_{0}^{1} H_{x_{i}}^{\prime}\left(X^{n}+\psi_{n}\right) d \lambda \\
& =H_{x_{i}}^{\prime}\left(X_{n}\right)+\int_{0}^{1} \sum_{j=1}^{4} \frac{1}{j !}\left(\psi_{n, 1} \frac{\partial}{\partial x_{1}}+\psi_{n, 2} \frac{\partial}{\partial x_{2}}+\cdots+\psi_{n, d} \frac{\partial}{\partial x_{d}}\right)^{j} H_{x_{i}}^{\prime}\left(X^{n}\right) d \lambda+R_{5},
\end{aligned}
$$

where

$$
\left(\psi_{n, 1} \frac{\partial}{\partial x_{1}}+\psi_{n, 2} \frac{\partial}{\partial x_{2}}+\cdots+\psi_{n, d} \frac{\partial}{\partial x_{d}}\right)^{j}=\sum_{r_{1}+r_{2}+\cdots+r_{d}=j} \frac{j !}{r_{1} ! r_{2} ! \cdots r_{d} !} \psi_{n, 1}^{r_{1}} \psi_{n, 2}^{r_{2}} \cdots \psi_{n, d}^{r_{d}} \frac{\partial^{j}}{\partial x_{1}^{r_{1}} \partial x_{2}^{r_{2}} \cdots \partial x_{d}^{r_{d}}},
$$

and

$$
R_{5}=\frac{1}{5 !} \int_{0}^{1} \int_{0}^{1}\left(\psi_{n, 1} \frac{\partial}{\partial x_{1}}+\psi_{n, 2} \frac{\partial}{\partial x_{2}}+\cdots+\psi_{n, d} \frac{\partial}{\partial x_{d}}\right)^{5} H_{x_{i}}^{\prime}\left(X^{n}+\mu \psi_{n}\right) d \mu d \lambda .
$$

Further, taking the Malliavin derivative $D_{s_{1} s_{2} s_{3}}^{\alpha}$ to $F_{x_{i}}^{X^{n}}$ with $\alpha=\left(j_{1}, j_{2}, j_{3}\right) \in \mathcal{A}_{3}$ implies

$$
\begin{aligned}
& \mathbb{E}_{t_{n}}^{X^{n}}\left[D_{s_{1} s_{2} s_{3}}^{\alpha}\left(F_{x_{i}}^{X^{n}}\right)\right]=\int_{0}^{1} \mathbb{E}_{t_{n}}^{X^{n}}\left[D_{s_{1} s_{2} s_{3}}^{\alpha} H_{x_{i}}^{\prime}\left(X^{n}+\psi_{n}\right)\right] d \lambda \\
= & D_{s_{1} s_{2} s_{3}}^{\alpha} H_{x_{i}}^{\prime}\left(X^{n}\right)+\int_{0}^{1} \sum_{j=1}^{4} \frac{1}{j !} \mathbb{E}_{t_{n}}^{X^{n}}\left[D_{s_{1} s_{2} s_{3}}^{\alpha}\left\{\left(\psi_{n, 1} \frac{\partial}{\partial x_{1}}+\psi_{n, 2} \frac{\partial}{\partial x_{2}}+\cdots+\psi_{n, d} \frac{\partial}{\partial x_{d}}\right)^{j} H_{x_{i}}^{\prime}\left(X^{n}\right)\right\}\right] d \lambda \\
& +\mathbb{E}_{t_{n}}^{X^{n}}\left[D_{s_{1} s_{2} s_{3}}^{\alpha}\left(R_{5}\right)\right] \\
= & \int_{0}^{1} \sum_{j=1}^{4} \sum_{r_{1}+r_{2}+\cdots+r_{d}=j} \frac{1}{r_{1} ! r_{2} ! \cdots r_{d} !} \mathbb{E}_{t_{n}}^{X^{n}}\left[D_{s_{1} s_{2} s_{3}}^{\alpha}\left\{\psi_{n, 1}^{r_{1}} \psi_{n, 2}^{r_{2}} \cdots \psi_{n, d}^{r_{d}}\right\}\right] \frac{\partial^{j+1} H}{\partial x_{i} \partial x_{1}^{r_{1}} \partial x_{2}^{r_{2}} \cdots \partial x_{d}^{r_{d}}}\left(X^{n}\right) d \lambda \\
& +D_{s_{1} s_{2} s_{3}}^{\alpha} H_{x_{i}}^{\prime}\left(X^{n}\right)+\mathbb{E}_{t_{n}}^{X^{n}}\left[D_{s_{1} s_{2} s_{3}}^{\alpha}\left(R_{5}\right)\right] .
\end{aligned}
$$

If $b, \sigma \in C_{b}^{3,6}, H \in C_{b}^{5}$, using (2.8) we have

$$
\mathbb{E}_{t_{n}}^{X^{n}}\left[D_{s_{1} s_{2} s_{3}}^{\alpha} F_{x_{i}}^{X^{n}}\right]=\omega_{\alpha}\left(t_{n}, X^{n}\right)+O_{n}(\Delta),
$$


where $\omega_{\alpha}\left(t_{n}, X^{n}\right)$ is a function depending only on the index $\alpha, t_{n}, b_{i}\left(t_{n}, X^{n}\right), \sigma_{i j}\left(t_{n}, X^{n}\right), H_{x_{i}}^{\prime}\left(X^{n}\right)$ $(1 \leqslant i, j \leqslant d)$, and their derivatives; the notation $O_{n}(\Delta)$ means that it has the estimate $\left|O_{n}(\Delta)\right| \leqslant C(1+$ $\left.\left|X^{n}\right|^{q}\right) \Delta$ with a prior known integer $q$ which does not depend on $n$. From $I_{(0,0,0), n}=\int_{t_{n}}^{t_{n+1}} \int_{t_{n}}^{s_{3}} \int_{t_{n}}^{s_{2}} d s_{1} d s_{2} d s_{3}=$ $\frac{1}{6} \Delta^{3}$, we obtain

$$
\begin{aligned}
& \sum_{i=1}^{d} \sum_{\alpha \in \mathcal{A}_{3}} I_{(0,0,0)}\left[\mathbb{E}_{t_{n}}^{X^{n}}\left[D_{s_{1} s_{2} s_{3}}^{\alpha}\left\{F_{x_{i}}^{X^{n}}\right\}\right]\right]_{t_{n}, t_{n+1}} g_{\alpha}^{i}\left(t_{n}, X^{n}\right) \\
= & \sum_{i=1}^{d} \sum_{\alpha \in \mathcal{A}_{3}}\left(\omega_{\alpha}\left(t_{n}, X^{n}\right)+O_{n}(\Delta)\right) g_{\alpha}^{i}\left(t_{n}, X^{n}\right) I_{(0,0,0), n} \\
= & \frac{1}{6} \Delta^{3} \sum_{i=1}^{d} \sum_{\alpha \in \mathcal{A}_{3}} \omega_{\alpha}\left(t_{n}, X^{n}\right) g_{\alpha}^{i}\left(t_{n}, X^{n}\right)+O_{n}\left(\Delta^{4}\right) .
\end{aligned}
$$

Then under the assumptions of the lemma, by the inequality (4.4a) in Hypothesis 4.3, it holds

$$
\begin{aligned}
& \frac{1}{6} \Delta^{3} \sum_{i=1}^{d} \sum_{\alpha \in \mathcal{A}_{3}}\left|\mathbb{E}_{t_{n}}^{X^{n}}\left[\omega_{\alpha}\left(t_{n}, X^{n}\right) g_{\alpha}^{i}\left(t_{n}, X^{n}\right)-\omega_{\alpha}\left(t_{n+1}, X^{n+1}\right) g_{\alpha}^{i}\left(t_{n+1}, X^{n+1}\right)\right]\right| \\
& \quad \leqslant C\left(1+\left|X^{n}\right|^{q}\right) \Delta^{4} .
\end{aligned}
$$

Under Hypothesis 4.3, from the equations (5.42) and the above inequality we obtain

$$
\begin{aligned}
\mid \sum_{i=1}^{d} \sum_{\alpha \in \mathcal{A}_{3}}\left\{I_{(0,0,0)}\left[\mathbb{E}_{t_{n}}^{X^{n}}\left[D_{s_{1} s_{2} s_{3}}^{\alpha}\left\{F_{x_{i}}^{X^{n}}\right\}\right]\right]_{t_{n}, t_{n+1}} g_{\alpha}^{i}\left(t_{n}, X^{n}\right)\right. \\
\left.\quad-I_{(0,0,0)}\left[\mathbb{E}_{t_{n}}^{X^{n}}\left[D_{s_{1} s_{2} s_{3}}^{\alpha}\left\{F_{x_{i}}^{X^{n+1}}\right\}\right]\right]_{t_{n+1}, t_{n+2}} g_{\alpha}^{i}\left(t_{n+1}, X^{n+1}\right)\right\} \mid \\
\leqslant C\left(1+\left|X^{n}\right|^{q}\right) \Delta^{4} .
\end{aligned}
$$

And under the assumption, it holds that

$$
\begin{aligned}
& \left|\sum_{i=1}^{d} \sum_{\alpha \in \mathcal{A}_{4}} I_{(0,0,0,0)}\left[\mathbb{E}_{t_{n}}^{X^{n}}\left[D_{s_{1} s_{2} s_{3} s_{4}}^{\alpha}\left\{F_{x_{i}}^{X_{n}}\right\} g_{\alpha}^{i}\left(s_{1}, X_{s_{1}}^{t_{n}, X^{n}}\right)\right]\right]_{t_{n}, t_{n+1}}\right| \\
& \quad \leqslant C\left(1+\left|X^{n}\right|^{q}\right) \Delta^{4} \\
& \quad \sum_{i=1}^{d} \sum_{\alpha \in \mathcal{A}_{4}} I_{(0,0,0,0)}\left[\mathbb { E } _ { t _ { n } } ^ { X ^ { n } } \left[D _ { s _ { 1 } s _ { 2 } s _ { 3 } s _ { 4 } } ^ { \alpha } \{ F _ { x _ { i } } ^ { X ^ { n + 1 } } \} g _ { \alpha } ^ { i } \left(s_{1}, X_{s_{1}}^{\left.\left.\left.t_{n+1}, X^{n+1}\right)\right]\right]_{t_{n+1}, t_{n+2}} \mid}\right.\right.\right. \\
& \quad \leqslant C\left(1+\left|X^{n}\right|^{q}\right) \Delta^{4} .
\end{aligned}
$$

Now combining the estimates (5.42), (5.43) and (5.44), we complete the proof.

Lemma 5.2.6. (See [30]) Let $\left(X_{r}^{t, x}, Y_{r}^{t, x}, Z_{r}^{t, x}\right)_{t \leqslant r \leqslant T}$ be the solution of (2.1), and let $R_{y 1}^{n}$ and $R_{z 1}^{n}$ be the truncation errors defined in (3.3) (3.7) for the $\mathrm{C}-\mathrm{N}$ scheme. If the terminal function $\varphi \in C_{b}^{4+\alpha}$ for some $\alpha \in(0,1), b, \sigma$ are bounded, $b, \sigma \in C_{b}^{2,4}$, and $f \in C_{b}^{2,4,4,4}$, then it holds that

$$
\begin{aligned}
& \mathbb{E}\left[\left|R_{y 1}^{N-1}\right|^{2}\right] \leqslant C\left(t_{N}-t_{N-1}\right)^{4}=C \Delta^{8}, \quad \mathbb{E}\left[\left|R_{z 1}^{N-1}\right|^{2}\right] \leqslant C\left(t_{N}-t_{N-1}\right)^{4}=C \Delta^{8}, \\
& \mathbb{E}\left[\left|R_{y 1}^{n}\right|^{2}\right] \leqslant C \Delta^{6}, \quad \mathbb{E}\left[\left|R_{z 1}^{n}\right|^{2}\right] \leqslant C \Delta^{6}, \quad 0 \leqslant n \leqslant N-2 .
\end{aligned}
$$

And if $\varphi \in C_{b}^{5+\alpha}$ for some $\alpha \in(0,1), b, \sigma \in C_{b}^{2,5}$ and $f \in C_{b}^{2,5,5,5}$, then it holds that

$$
\mathbb{E}\left[\left|\nabla_{x^{n}} R_{y 1}^{n}\right|^{2}\right] \leqslant C \Delta^{6}, \quad \mathbb{E}\left[\left|\nabla_{x^{n}} R_{z 1}^{n}\right|^{2}\right] \leqslant C \Delta^{6}, \quad 0 \leqslant n \leqslant N-2 .
$$

Here $C$ is a generic positive constant depending on $K$, the initial condition of $X_{t}$, and upper bounds of the derivatives of $b, \sigma, f$ and $\varphi$.

The above lemma can be proved by using the Taylor and Itô-Taylor expansion. Here we omit the proof. Please see the details in [30]. Now combining Lemmas 5.2.15 5.2.5, we state our truncation error estimates in the following lemma. 
Lemma 5.2.7. For $X^{n+1}=\sum_{\alpha \in \Gamma_{2}} g_{\alpha}\left(t_{n}, X^{n}\right) I_{\alpha, n}$, if $b, \sigma \in C_{b}^{2,5}, f(t, X, Y, Z) \in C_{b}^{3,5,5,5}$ and $\varphi \in C_{b}^{6+\alpha}$ for some $\alpha \in(0,1)$, then under Hypotheses 4.1 and 4.3 , there exists a generic positive integer $q$ such that

$$
\begin{aligned}
& \max _{0 \leqslant n \leqslant N-2}\left|\nabla_{x^{n}} R_{y 2}^{n}\right| \leqslant C\left(1+\left|X^{n}\right|^{q}\right) \Delta^{3}, \\
& \max _{0 \leqslant n \leqslant N-2}\left|\nabla_{x^{n}} R_{z 2}^{n}\right| \leqslant C\left(1+\left|X^{n}\right|^{q}\right) \Delta^{3},
\end{aligned}
$$

and

$$
\begin{aligned}
& \max _{0 \leqslant n \leqslant N-2}\left|\mathbb{E}_{t_{n}}^{X^{n}}\left[R_{y 1}^{n+1} \Delta W_{n}^{\top}\right]\right| \leqslant C\left(1+\left|X^{n}\right|^{q}\right) \Delta^{4}, \\
& \max _{0 \leqslant n \leqslant N-2}\left|\mathbb{E}_{t_{n}}^{X^{n}}\left[\Delta W_{n} \nabla_{x^{n}} R_{y 1}^{n+1}\right]\right| \leqslant C\left(1+\left|X^{n}\right|^{q}\right) \Delta^{4}, \\
& \max _{0 \leqslant n \leqslant N-2}\left|\mathbb{E}_{t_{n}}^{X^{n}}\left[R_{y 2}^{n+1} \Delta W_{n}^{\top}\right]\right| \leqslant C\left(1+\left|X^{n}\right|^{q}\right) \Delta^{4}, \\
& \max _{0 \leqslant n \leqslant N-2}\left|\mathbb{E}_{t_{n}}^{X^{n}}\left[\Delta W_{n} \nabla_{x^{n}} R_{y 2}^{n+1}\right]\right| \leqslant C\left(1+\left|X^{n}\right|^{q}\right) \Delta^{4} .
\end{aligned}
$$

And if $b, \sigma \in C_{b}^{2,5}, f(t, X, Y, Z) \in C_{b}^{3,6,6,6}$ and $\varphi \in C_{b}^{7+\alpha}$ for some $\alpha \in(0,1)$, then

$$
\begin{aligned}
& \max _{0 \leqslant n \leqslant N-2}\left|R_{z 1}^{n}-\mathbb{E}_{t_{n}}^{X^{n}}\left[R_{z 1}^{n+1}\right]\right| \leqslant C\left(1+\left|X^{n}\right|^{q}\right) \Delta^{4}, \\
& \max _{0 \leqslant n \leqslant N-2}\left|R_{z 2}^{n}-\mathbb{E}_{t_{n}}^{X^{n}}\left[R_{z 2}^{n+1}\right]\right| \leqslant C\left(1+\left|X^{n}\right|^{q}\right) \Delta^{4} .
\end{aligned}
$$

Here $C$ is a generic positive constant depending on $K$, and upper bounds of the derivatives of $b, \sigma, f$ and $\varphi$.

Proof. Under the conditions of the lemma, by the Feynman-Kac formula (1.2), the solution $\left(Y_{t}^{t_{n}, X^{n}}, Z_{t}^{t_{n}, X^{n}}\right)$ of FBSDEs (2.1) can be represented as

$$
Y_{t}^{t_{n}, X^{n}}=u\left(t, X_{t}^{t_{n}, X^{n}}\right), \quad Z_{t}^{t_{n}, X^{n}}=u_{x}\left(t, X_{t}^{t_{n}, X^{n}}\right) \sigma\left(t, X_{t}^{t_{n}, X^{n}}\right), \forall t \in[0, T),
$$

where $u(t, x)$ satisfies the parabolic PDE (1.3). According to (5.50), we set

$$
\begin{aligned}
H\left(X_{t_{n+1}}^{t_{n}, X^{n}}\right)= & \mathbb{E}_{t_{n}}^{X^{n}}\left[Y_{t_{n+1}}^{t_{n}, X^{n}}\right]+\frac{1}{2} \Delta \mathbb{E}_{t_{n}}^{X^{n}}\left[f_{t_{n+1}}^{t_{n}, X^{n}}\right] \\
= & \mathbb{E}_{t_{n}}^{X^{n}}\left[u\left(t_{n+1}, X_{t_{n+1}}^{t_{n}, X^{n}}\right)\right] \\
& +\frac{1}{2} \Delta \mathbb{E}_{t_{n}}^{X^{n}}\left[f\left(t_{n+1}, X_{t_{n+1}}^{t_{n}, X^{n}}, u\left(t_{n+1}, X_{t_{n+1}}^{t_{n}, X^{n}}\right), u_{x}\left(t_{n+1}, X_{t_{n+1}}^{t_{n}, X^{n}}\right) \sigma\left(t_{n+1}, X_{t_{n+1}}^{t_{n}, X^{n}}\right)\right)\right]
\end{aligned}
$$

and

$$
\begin{aligned}
H\left(X^{n+1}\right)= & \mathbb{E}_{t_{n}}^{X^{n}}\left[Y_{t_{n+1}}^{t_{n+1}, X^{n+1}}\right]+\frac{1}{2} \Delta \mathbb{E}_{t_{n}}^{X^{n}}\left[f_{t_{n+1}}^{t_{n+1}, X^{n+1}}\right] \\
= & \mathbb{E}_{t_{n}}^{X}\left[u\left(t_{n+1}, X^{n+1}\right)\right] \\
& +\frac{1}{2} \Delta \mathbb{E}_{t_{n}}^{X^{n}}\left[f\left(t_{n+1}, X^{n+1}, u\left(t_{n+1}, X^{n+1}\right), u_{x}\left(t_{n+1}, X^{n+1}\right) \sigma\left(t_{n+1}, X^{n+1}\right)\right)\right] .
\end{aligned}
$$

Then,

$$
R_{y 2}^{n}=H\left(X_{t_{n+1}}^{t_{n}, X^{n}}\right)-H\left(X^{n+1}\right) .
$$

By the theory of partial differential equations [9], under the conditions of the lemma, it is easy to check that the function $H$ satisfies the conditions in Lemma 5.2.1, thus we have the estimate $\nabla_{x^{n}} R_{y 2}^{n}$ in (5.47). Similarly under the conditions of this lemma, we have the estimates in (5.48) and (5.49) by using Lemma 5.2 .25 .2 .5 . The proof is completed.

Lemma 5.2.8. Assume $Y^{N}=\varphi\left(X^{N}\right)$. Under Hypothesis 4.3 and the conditions of Lemma 5.2.6, it holds that

$$
\mathbb{E}\left[\left|e_{Y}^{N-1}\right|^{2}\right] \leqslant C \Delta^{4}, \quad \mathbb{E}\left[\left|e_{Z}^{N-1}\right|^{2}\right] \leqslant C \Delta^{4}, \quad \mathbb{E}\left[\left|e_{\nabla Y}^{N-1}\right|^{2}\right] \leqslant C \Delta^{4}, \quad \mathbb{E}\left[\left|e_{\nabla Z}^{N-1}\right|^{2}\right] \leqslant C \Delta^{4} .
$$

where $C$ is a generic constant depending on $K, L$, the initial condition of $X_{t}$, and upper bounds of derivatives of $b, \sigma, f$ and $\varphi$.

Proof. We know that for weak order-2 scheme (2.4), Hypothesis 4.3 holds true with $\beta=\gamma=2$ (readers can refer to Section 4.3.2 in 32 for the detailed proof). Combining with Lemma 5.2.6 we get

$$
\sum_{j=1}^{2} \mathbb{E}\left[\left|R_{y j}^{N-1}\right|^{2}\right] \leqslant C \Delta^{8}, \quad \sum_{j=1}^{2} \mathbb{E}\left[\left|R_{z j}^{N-1}\right|^{2}\right] \leqslant C \Delta^{8} .
$$


By the equalities (3.3), (3.6) and (3.9), we deduce

$$
\mathbb{E}\left[\left|e_{Z}^{N-1}\right|^{2}\right]=\frac{1}{\Delta^{2}} \mathbb{E}\left[\left|\sum_{j=1}^{2} R_{z j}^{N-1}\right|^{2}\right] \leqslant \frac{1}{\Delta^{2}} \sum_{j=1}^{2} \mathbb{E}\left[\left|R_{z j}^{N-1}\right|^{2}\right] \leqslant C \Delta^{4}
$$

and

$$
\mathbb{E}\left[\left|e_{Y}^{N-1}\right|^{2}\right] \leqslant C \Delta^{2} \mathbb{E}\left[\left|e_{Y}^{N-1}\right|^{2}+\left|e_{Z}^{N-1}\right|^{2}\right]+C \sum_{j=1}^{2} \mathbb{E}\left[\left|R_{y j}^{N-1}\right|^{2}\right] \leqslant C \Delta^{2} \mathbb{E}\left[\left|e_{Y}^{N-1}\right|^{2}\right]+C \Delta^{4},
$$

which implies $\mathbb{E}\left[\left|e_{Y}^{N-1}\right|^{2}\right] \leqslant \frac{C \Delta^{4}}{1-C \Delta^{4}} \leqslant C \Delta^{4}$. Similarly we can prove $\mathbb{E}\left[\left|e_{\nabla Y}^{N-1}\right|^{2}\right] \leqslant C \Delta^{4}$ and $\mathbb{E}\left[\left|e_{\nabla Z}^{N-1}\right|^{2}\right] \leqslant$ $C \Delta^{4}$. The proof is completed.

\subsubsection{Proof of Theorem 4.2}

After the above preparations, we now give the proof of Theorem 4.2 as follows.

Proof. Under the conditions of the theorem, if Hypothesis 4.3 holds, we have

$$
\mathbb{E}\left[\left|X^{n}\right|^{q}\right] \leqslant C\left(1+\mathbb{E}\left[\left|X_{0}\right|^{q}\right]\right)
$$

then according to Lemmas 5.2.6 5.2.8, we obtain the estimates

$$
\begin{gathered}
\mathbb{E}\left[\left|e_{Y}^{N-1}\right|^{2}+\left|e_{Z}^{N-1}\right|^{2}+\left|e_{\nabla Y}^{N-1}\right|^{2}+\left|e_{\nabla Z}^{N-1}\right|^{2}\right] \leqslant C \Delta^{4}, \\
\max _{0 \leqslant n \leqslant N-2} \sum_{j=1}^{2} \mathbb{E}\left[\left|R_{y j}^{n}\right|^{2}+\left|\nabla_{x^{n}} R_{y j}^{n}\right|^{2}+\left|\nabla_{x^{n}} R_{z j}^{n}\right|^{2}+\left|\mathbb{E}_{t_{n}}^{X^{n}}\left[R_{y j}^{n+1}\right]\right|^{2}+\left|\mathbb{E}_{t_{n}}^{X^{n}}\left[R_{z j}^{n+1}\right]\right|^{2}\right. \\
\left.+\left|\mathbb{E}_{t_{n}}^{X^{n}}\left[\nabla_{x^{n}} R_{y j}^{n+1}\right]\right|^{2}+\left|\mathbb{E}_{t_{n}}^{X^{n}}\left[\nabla_{x^{n}} R_{z j}^{n+1}\right]\right|^{2}+\left|\mathbb{E}_{t_{n}}^{X^{n}}\left[\nabla_{x^{n+1}} R_{z j}^{n+1}\right]\right|^{2}\right] \leqslant C \Delta^{6}, \\
\max _{0 \leqslant n \leqslant N-2} \sum_{j=1}^{2} \mathbb{E}\left[\left|\mathbb{E}_{t_{n}}^{X^{n}}\left[\Delta W_{n} \nabla_{x^{n}} R_{y j}^{n+1}\right]\right|^{2}+\left|\mathbb{E}_{t_{n}}^{X^{n}}\left[R_{y j}^{n+1} \Delta W_{n}^{\top}\right]\right|^{2}+\left|R_{z j}^{n}-\mathbb{E}_{t_{n}}^{X^{n}}\left[R_{z j}^{n+1}\right]\right|^{2}\right] \\
\leqslant C \Delta^{8} .
\end{gathered}
$$

Inserting the above estimates into (4.12) in Theorem 4.1, we easily deduce (4.13). The proof is completed.

\section{Conclusions}

In this paper, we considered the theoretical error estimates of the Crank-Nicolson (C-N) scheme for solving decoupled FBSDEs proposed in 29. By properly using the Young's inequality to the error equations of the C-N scheme and their associated variational equations, we first rigorously obtained a general error estimate result for the $\mathrm{C}-\mathrm{N}$ scheme. This result also implies the stability of the scheme. Then by the Taylor and Itô-Taylor expansions, the theory of multiple Malliavin calculus, and the local truncation error cancelation techniques, we theoretically obtained the truncation error estimates of the scheme. Finally based on the general error estimate result and the truncation error estimates, we theoretically proved that the accuracy of the C-N scheme for solving decoupled FBSDEs is of second order.

Acknowledgements This author's research is partially supported by the National Natural Science Foundations of China under grant numbers 11426152, 11501366 and 11571206.

\section{References}

1 F. Antonelli, Backwardforward stochastic differential equations, Ann. Appl. Probab., 3 (1993), pp. 777-793.

2 C. Bender And R. Denk, A forward scheme for backward SDE, Stochastic Process. Appl., 117 (2007), pp. $1793-1812$.

3 C. Bender and J. Zhang, Time discretization and Markovian iteration for coupled FBSDEs, Ann. Appl. Probab., 18, 1 (2008), pp. 143-177.

4 B. Bouchard and N. Touzi, Discrete-time approximation and Monte-Carlo simulation of backward stochastic differential equations, Stochastic Process. Appl., 111 (2004), pp. 175-206.

5 D. CRisan And K. Manolarakis, Second order discretization of backward SDE and simulation with the cubature method, Ann. Appl. Probab., 24, 2 (2014), pp. 652-678.

6 J. Cvitanic and J. Zhang, The steepest descent method for forward-backward SDE, Electron. J. Probab. 10 (2005), pp. $1468-1495$. 
7 F. Delarue and S. Menozzi, A forward-backward stochastic algorithm for quasi-linear PDEs, Ann. Appl. Probab. 16 (2006), pp. 140-184.

8 J. Douglas, J. Ma and P. Protter, Numerical methods for forward-backward stochastic differential equations, Ann. Appl. Probab. 6 (1996), pp. 940-968.

9 L. C. Evans, Partial Differential Equations, Providence, RI, American Mathematical Society, 1998.

10 E. Gobet, C. Labart, Error expansion for the discretization of backward stochastic differential equations, Stochastic Process. Appl., 117 (2007), pp. 803-829.

11 E. Gobet, J. P. Lemmor And X. WARIn, A regression-based Monte Carlo method to solve backward stochastic differential equations, Ann. Appl. Probab., 15 (2005), pp. 2172-2202.

12 N. El. Karoui, S. Peng, and M. C. Quenez, Backward stochastic differential equations in finance, Math. Financ., 7 (1997), pp. 1-71.

13 P. E. Kloeden and E. Platen, Numerical Solution of Stochastic Differential Equations, Springer-Verlag, Berlin, 1992.

14 O. Ladyzenskaja, V. Solonnikov, and N. Uralceva, Linear and Quasilinear Equations of Parabolic Type, Providence, RI, American Mathematical Society, 1968.

15 J. Ma, P. Protter, J. M. San, And S. Torres, Numerical methods for backward stochastic differential equations, Ann. Appl. Probab., 12 (2002), pp. 302-316.

16 J. Ma, J. Shen, And Y. Zhao, On numerical approximations of forward-backward stochastic differential equations, SIAM J. Numer. Anal., 46 (2008), pp. 2636-2661.

17 J. Ma And J. Yong, Forward-Backward Stochastic Differential Equations and Their Applications, Lecture Notes in Mathematics, 1702. Springer-Verlag, Berlin, 1999.

18 D. Nualart, The Malliavin Calculus and Related Topics, Springer Verlag, Berlin, 1995.

19 E. Pardoux and S. Peng, Adapted solution of a backward stochastic differntial equation, Systems Control Lett., 14 (1990), pp. 55-61.

20 E. Pardoux And A. Rascanu, Stochastic Differential Equations, Backward SDEs, Partial Differential Equations, Springer, 2014.

21 S. PEng, A general stochastic maximum principle for optimal control problems, SIAM J. Control Optim., 28 (1990), pp. 966-979.

22 S. Peng, Probabilistic interpretation for systems of quasilinear parabolic partial differential equations, Stoch. Stoch. Rep., 37 (1991), pp. 61-74.

23 S. Peng, Backward SDE and Related g-Expectation, in Backward stochastic differential equations (Paris, 1995-1996), Pitman Res. Notes Math. Ser., 364, Longman, Harlow, UK, 1997, pp. 141-159.

24 J. Zhang, A numerical scheme for BSDE, Ann. Appl. Probab., 14 (2004), pp. 459-488.

25 W. Zhao, L. Chen, and S. Peng, A new kind of accurate numerical method for backward stochastic differential equations, SIAM J. Sci. Comput., 28 (2006), pp. 1563-1581.

26 W. Zhaо, Y. Fu, And T. Zhou, New kinds of high-order multi-step schemes for coupled forward backward stochastic differential equations, SIAM J. Sci. Comput., 36, 4 (2014), pp. A1731-A1751.

27 W. Zhao, Y. Li, And G. Zhang, A generalized $\theta$-scheme for solving backward stochastic differential equations, Discrete Contin. Dyn. Syst. Ser. B, 17, 5 (2012), pp. 1585-1603.

28 W. ZhaO, Y. Li, AND L. Ju, Error estimates of the Crank-Nicolson scheme for solving backward stochastic differential equations, Int. J. Numer. Anal. Model., 10, 4 (2013), pp. 876-898.

29 W. Zhao, Y. Li, AND Y. Fu, Second-order schemes for solving decoupled forward backward stochastic differential equations, Sci. China Math., 57, 4 (2014), pp. 665-686.

30 W. Zhao, J. WAng, And S. Peng, Error estimates of the $\theta$-scheme for backward stochastic differential equations, Discrete Contin. Dyn. Syst. Ser. B, 12, 4 (2009), pp. 905-924.

31 W. Zhao, G. Zhang, And L. Ju, A stable multistep scheme for solving backward stochastic differential equations, SIAM J. Numer. Anal., 2010, 48, 4 (2010), pp. 1369-1394.

32 W. Zhao, W. Zhang, and L. Ju, A numerical method and its error estimates for the decoupled forward-backward stochastic differential equations, Commun. Comput. Phys., 15 (2014), pp. 618-646. 